\title{
Station and Transect Data for Mermaid (Rowley Shoals), Scott and Seringapatam Reefs, Western Australia.
}

\author{
Clay Bryce \\ Department of Aquatic Zoology, Western Australian Museum, Locked Bag 49, Welshpool, D.C. 6986. \\ Email: clay.bryce@museum.wa.gov.au
}

The biodiversity survey of Mermaid (Rowley Shoals), Scott and Seringapatam Reefs, Western Australia, undertaken in September 2006, recorded six faunal taxa (Porifera, Scleracterinia corals, Crustacea, Molluscs, Echinodermata and Pisces (fish) as well as the marine flora (algae and seagrasses).

Stations were sited to represent as many habitats as possible. Where practical, stations were overlayed or placed close to established Australian Institute of Marine Science (AIMS) monitoring sites (A. Heyward, pers. comm.) and Western Australian Museum (WA Museum) collecting sites as cited in Berry (1986) (Table 1). Five stations were also located in areas proposed for industrial activity (stations 25 and 28 from South Scott Reef; stations 31, 32 and 35 from North Scott Reef).

\section{STATION SITES AND TRANSECT DATA:}

(Figures 1, 2 and 3; Table 2, 3, 4)

Location of station sites and transect data for Mermaid (Rowley Shoals), Scott and Seringapatam Reefs can be found in Figure 1 and Table 2; Figure 2 and Table 3 and; Figure 3 and Table 4 respectively.

Tables 2, 3 and 4 provide station numbers, coordinates, survey dates, transect data (T- depth, T-length, T-width, T-area in $\mathrm{m}^{2}$ and hectares) and a brief habitat descriptor for the surveyed reefs. A total of 45 stations were surveyed (Mermaid 17 stations; South Scott Reef 14; North Scott Reef 10 and Seringapatam 5) over 16 sampling days. The station identification data (date, habitat descriptor, station number, coordinates in UTM, depth and decimal latitude and longitude) are in bold type for easy recognition.

The coordinates for stations and transects were recorded in the Universal Transverse Mercator geographic coordinate system (UTM), which uses "northings" and "eastings". These coordinates were converted to latitude and longitude (degrees and decimal minutes) for the convenience of reader use. The identification waypoint for stations was determined at the deepest point of each station. Within each subtidal station two sets of bisecting transect were established. This approach helped to alleviate site crowding with 10 divers in the water at any one time. It also allowed for predetermined sampling methodologies for each taxa to be accommodated within the one hour dive time. Intertidal stations were sampled qualitatively for all taxa, except for Porifera.

The set of transects dedicated to Algae (A), Mollusca (M), Echinodermata (E) and Fish (F) ran from the deepest point (station waypoint) to the shallowest part of each station. The fish transects (F1) ran in a general upslope or cross-station direction and had no recorded end waypoint but concluded at the reef edge. This set of transects crossed (i.e. ran perpendicular to) the second set, which were recording Scleracterinia (S), Porifera $(\mathrm{P})$ and Crustacea $(\mathrm{C})$. These latter transects were depth restricted at approximately $5 \mathrm{~m}$ and $12 \mathrm{~m}$ (relative to mean sea level). When they were sited at a significant distance from the station waypoint a decimal latitude and longitude conversion was also provided in Tables 2, 3 and 4. The area encompassed by these transects was recorded on video and analysed for percentage cover (Morrison, P., this volume).

Distances defining transect lengths were determined from measuring tape and way point coordinates. For the A, M and E transects, where a slope distance was required, a simple straight line estimate was calculated. The slope calculation used is expressed as:

$\sqrt{ } a^{2}+b^{2}+c^{2}$ Where:

- $\mathrm{a}=$ "easting" coordinate (UTM) distance between deepest buoy and shallowest buoy

- $\mathrm{b}=$ "northing" coordinate (UTM) distance between deepest buoy and shallowest buoy

- $\mathrm{c}=$ difference in the depths at the two buoys.

- Detailed methodologies for each taxon are contained within the following papers of this volume.

\section{To read the tables:}

Example 1: Station 17, Scott Reef.

On the 18 September 2006 at Scott Reef dive 


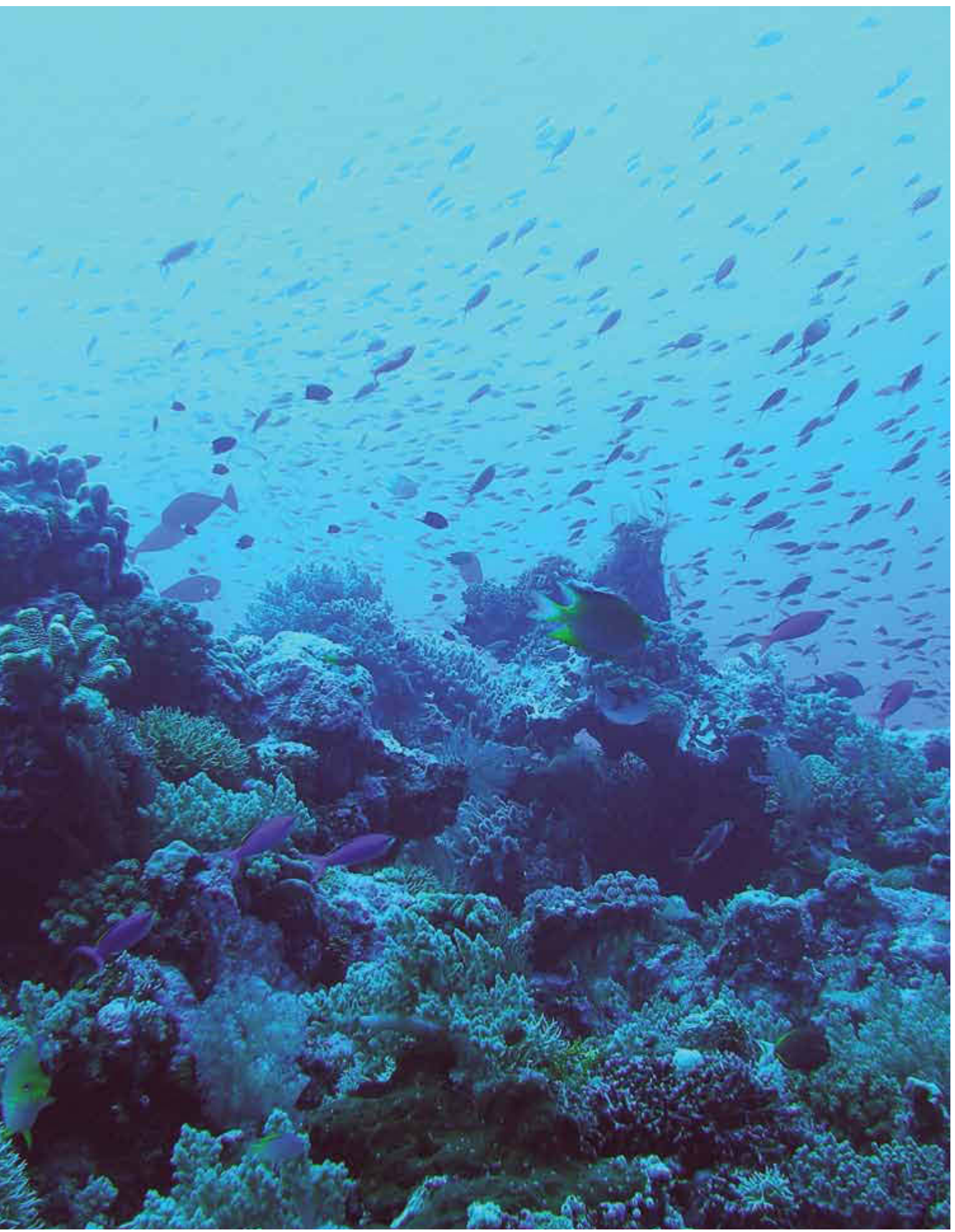

Above: Station 2, Mermaid Reef. (Photo: Sue Morrison) 
Table 1 Stations with matching coordinates for the 2006 WA Museum survey, AIMS monitoring stations and WA Museum stations (Berry, 1986).

\begin{tabular}{l|l|l}
\hline $\begin{array}{l}\text { WA Museum Stations } \\
\text { (This survey - 2006) }\end{array}$ & $\begin{array}{l}\text { WA Museum Stations } \\
\text { (Berry, 1986) }\end{array}$ & AIMS Monitoring Stations \\
\hline 11 & & RL1-1(old) (Mermaid) \\
\hline 18 and 24 & 2 and 4 (Scott) & \\
\hline 22 & $23($ Scott) & \\
\hline 26 & $20($ Scott) & \\
\hline 27 & $19($ Scott) & \\
\hline 26 & & SL2-1 (Scott) \\
\hline 30 & & SS1-1 (Scott) \\
\hline 34 & & SL4-1 (Scott) \\
\hline 41 & 13 (Seringapatam) & \\
\hline 44 and 45 & & SS3 (Seringapatam) \\
\hline
\end{tabular}

Station 17 (located at UTM Zone 50S, easting 369212 : northing 8429152 or $\left.14^{\circ} 12.360^{\prime} \mathrm{S}: 121^{\circ} 47.273^{\prime} \mathrm{E}\right)$ a 1 m wide transect for Algae (AI, A2), Mollusca (M1, M2), Echinodermata (E1, E2) and Fish (F1) began in $20 \mathrm{msw}$ running for $72 \mathrm{~m}$ and ended in $3.5 \mathrm{msw}$ at coordinates $14^{\circ} 12.325^{\prime} \mathrm{E}$ : $121^{\circ} 47.289^{\prime} \mathrm{E}$. The survey area covered was $72 \mathrm{~m}^{2}$ or 0.007 ha. Note that the Fish transect (F1) end coordinate was approximate and not recorded.

The start points for the transects of Porifera (P1 at $10.5 \mathrm{~m}$ and P2 at $3.5 \mathrm{~m}$ ), Scleracterinia corals (S1 at $10.5 \mathrm{~m}$ and S2 at $3.5 \mathrm{~m}$ ) and Crustacea (C1 at $10.5 \mathrm{~m}$ and $\mathrm{C} 2$ at $3.5 \mathrm{~m}$ ) are recorded in UTMs ("northings' and "eastings"). The location of this set of replicated transects was also converted to decimal latitude and longitude (14²12.343'S: $121^{\circ} 47.281^{\prime} \mathrm{E}$ to $14^{\circ} 12.325^{\prime} \mathrm{S}$ : $\left.121^{\circ} 47.289^{\prime} \mathrm{E}\right)$ for convenience as they were considered to be at a significant distance from the identifying station marker located at $14^{\circ} 12.360^{\prime}$ S: $121^{\circ} 47.273^{\prime} \mathrm{E}$.

\section{Example 2: Station 21, Scott Reef.}

On the 20 September 2006 at Scott Reef intertidal Station 21 (located at UTM Zone 50S easting 364563 : northing 8443446 or $14^{\circ} 04.593^{\prime} \mathrm{S}$ : $\left.121^{\circ} 44.732^{\prime} \mathrm{E}\right)$ a 1 $\mathrm{m}$ wide transect for Porifera (P1) began in $0 \mathrm{msw}$ running for a measured $15 \mathrm{~m}$. The survey area covered was $15 \mathrm{~m}^{2}$ or 0.002 ha. All other marine groups were surveyed qualitatively.

\section{REFERENCE:}

Berry, P.F. and Marsh, L.M. (1986). Part 1. History of investigation and description of physical environment. In Berry. P.F. (ed.), Faunal Surveys of the Rowley Shoals, Scott Reef, and Seringapatam Reef, North-western Australia. Records of the Western Australian Museum, Supplement 25: 1-25.

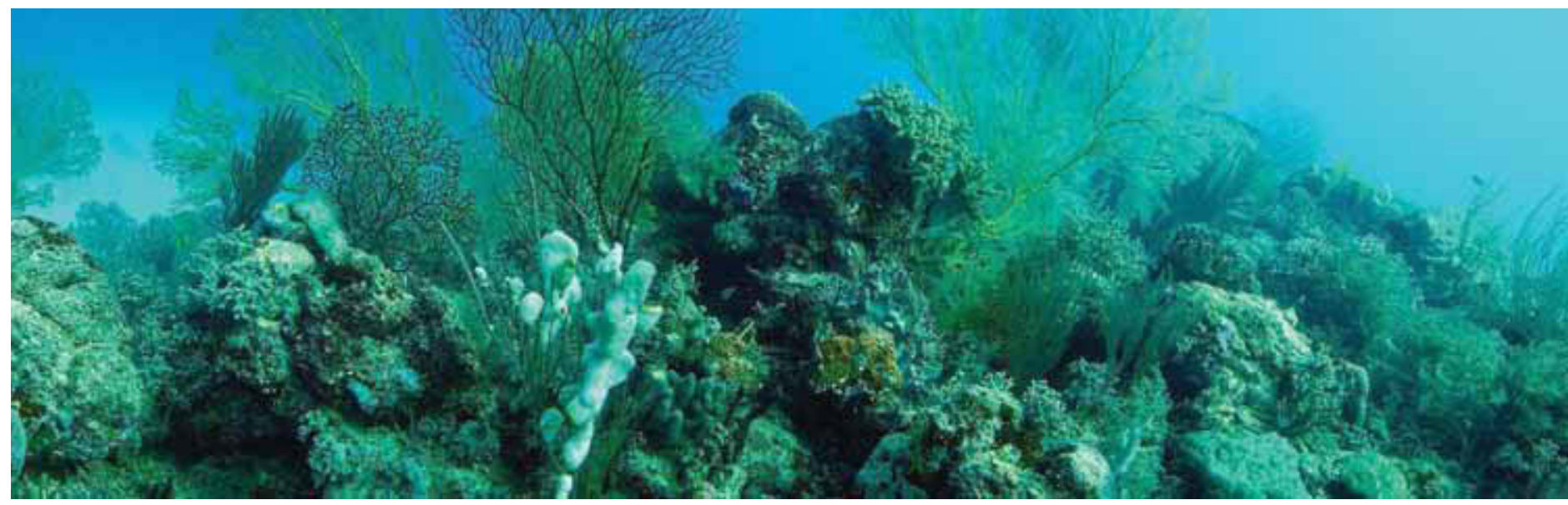

Above: Station 34, North Scott Reef. Numerous sea fans indicate a periodical, strong current flow. (Photo: John Huisman) 


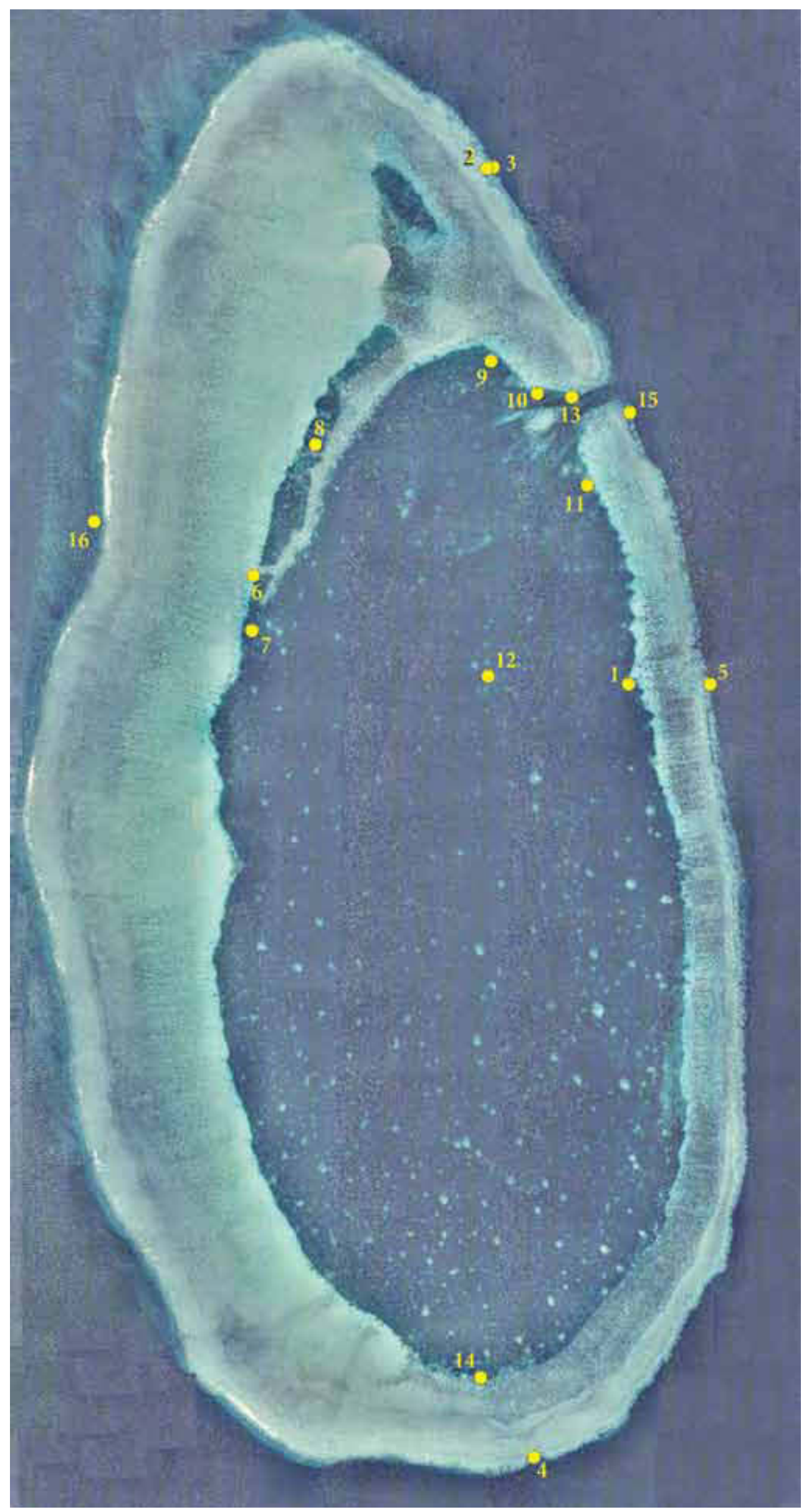

Figure 1 Mermaid Reef (Rowley Shoals) with surveyed station sites (2006) 


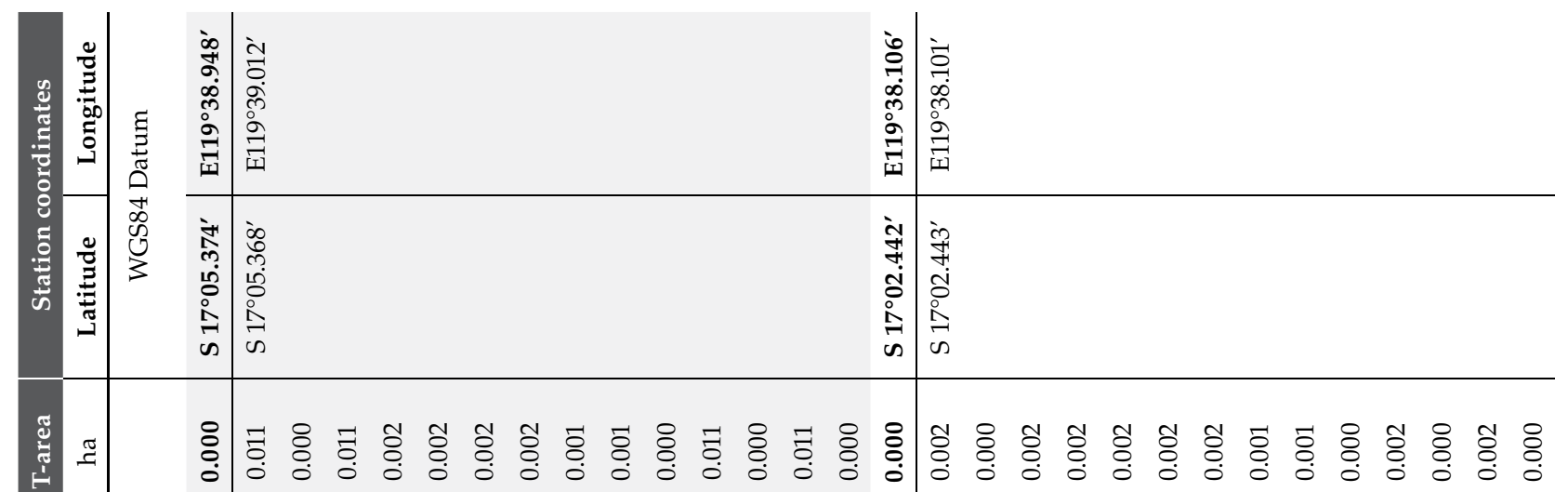

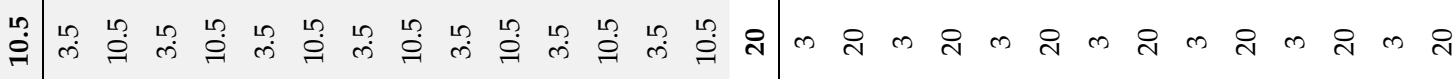

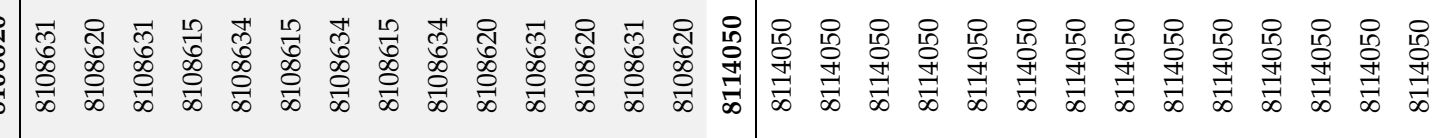

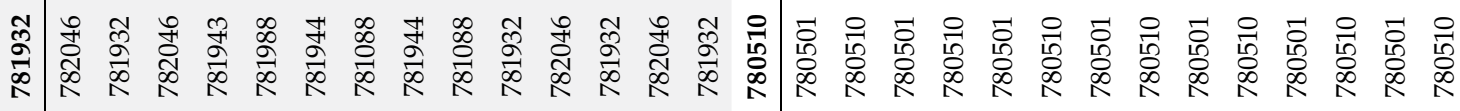

$\frac{\pi}{\pi}$

完
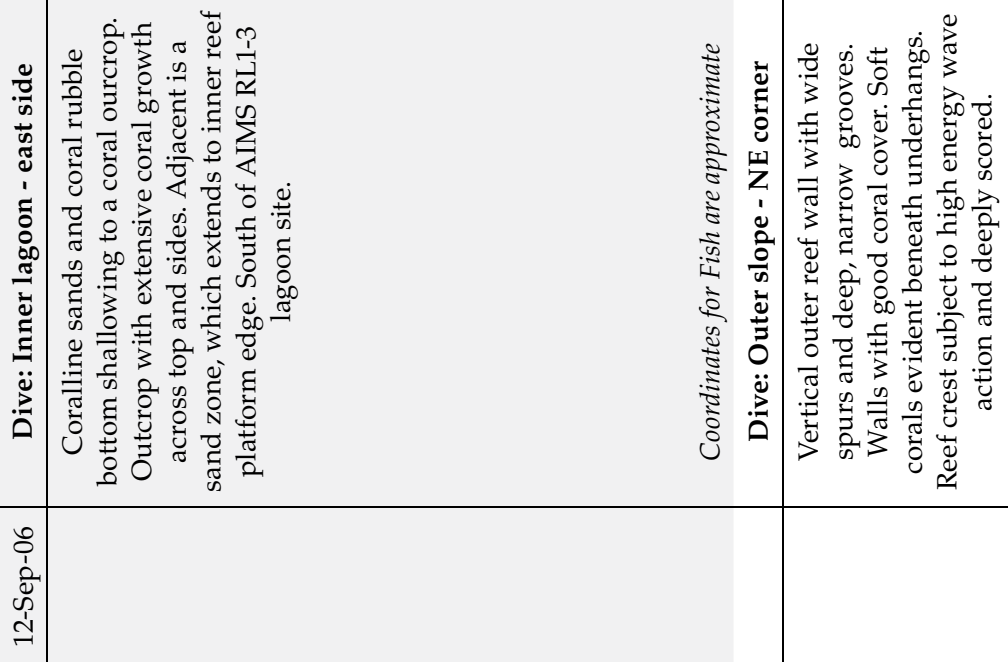


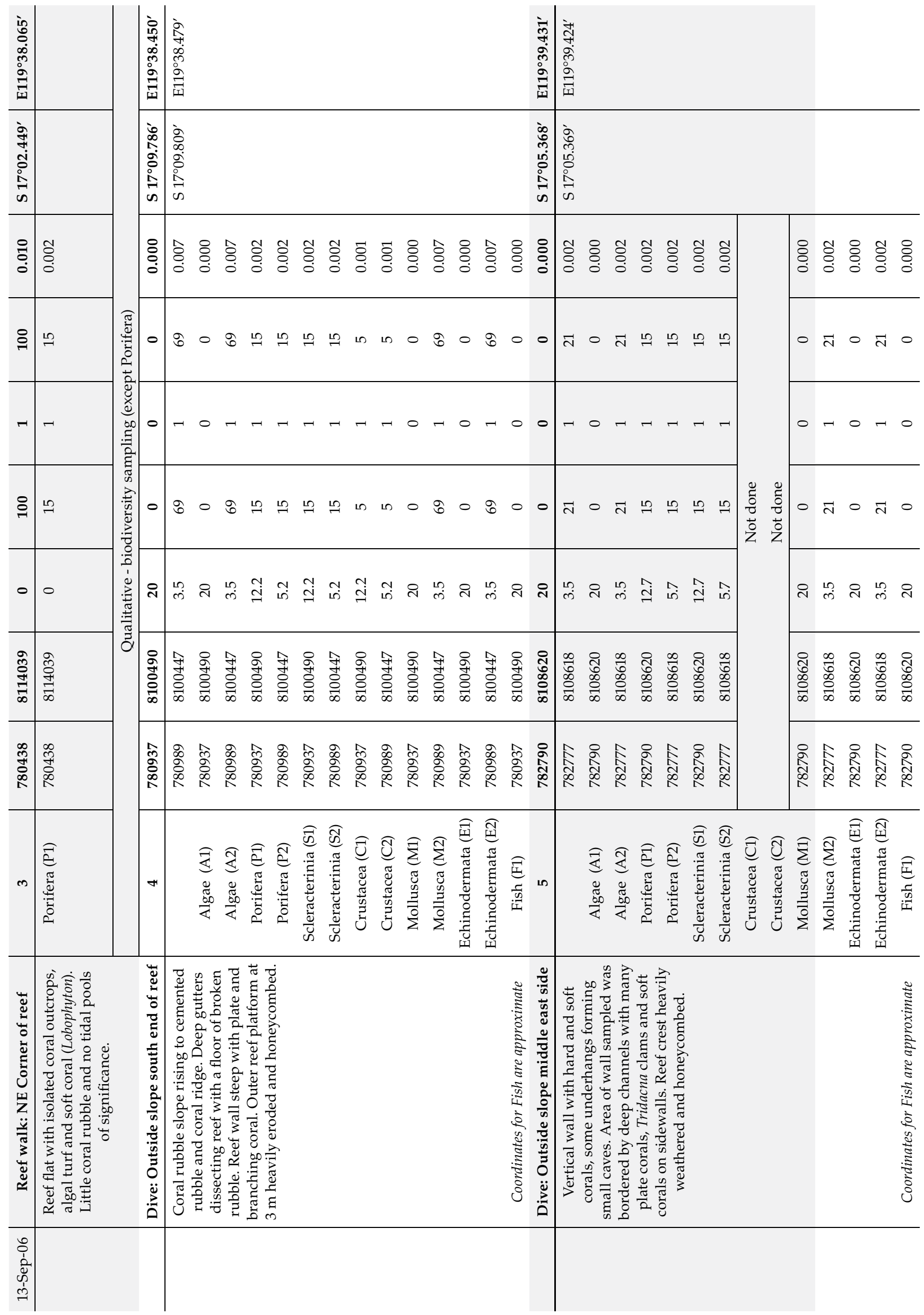




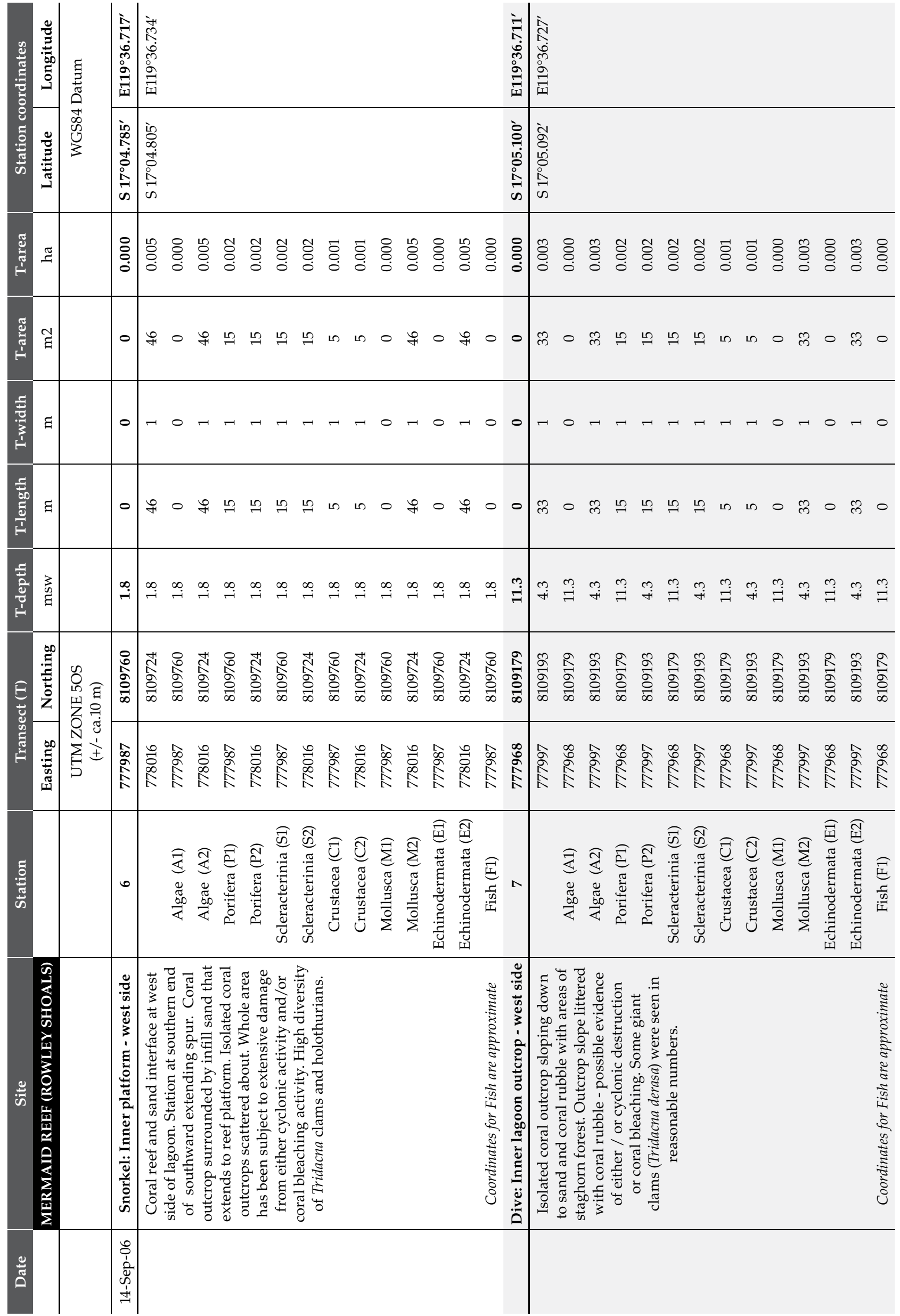




\begin{tabular}{|c|c|c|c|c|c|}
\hline & 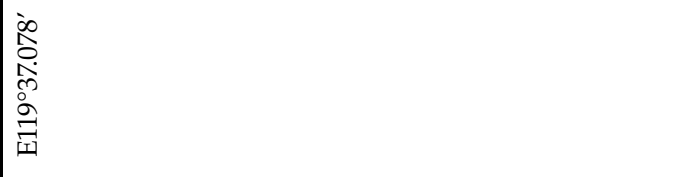 & 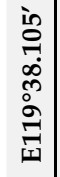 & 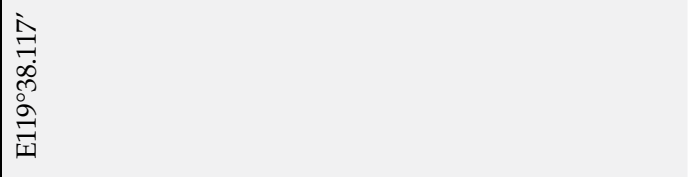 & 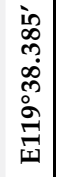 & 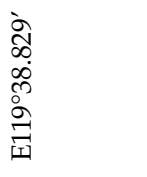 \\
\hline & & 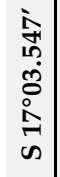 & \begin{tabular}{|l}
10 \\
0 \\
10 \\
$\infty$ \\
0 \\
0 \\
1 \\
$\omega$ \\
$\omega$
\end{tabular} & 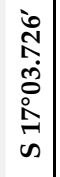 & 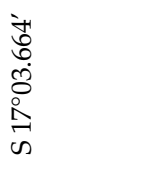 \\
\hline & 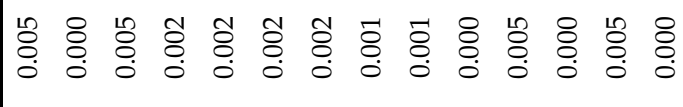 & $\stackrel{0}{0}$ & 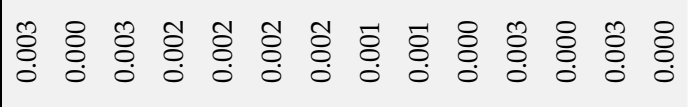 & $\begin{array}{l}0 \\
\vdots \\
\vdots\end{array}$ & 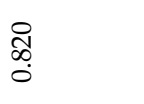 \\
\hline & L & 0 & 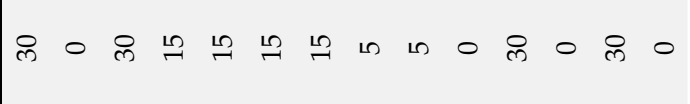 & 0 & ¿্ి \\
\hline D & $r \quad 0-r-r-r-r \quad r_{-r} 0-10$ & 0 & $r 0 r-r-r-r-r^{-}$ & $\circ$ & ? \\
\hline & 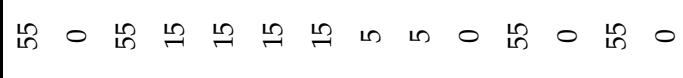 & 0 & 品 & 0 & శ్ \\
\hline & 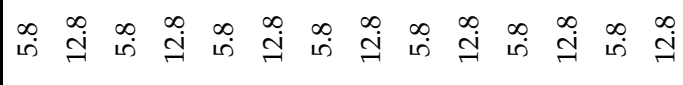 & $\underset{\vec{E}}{\vec{F}}$ & 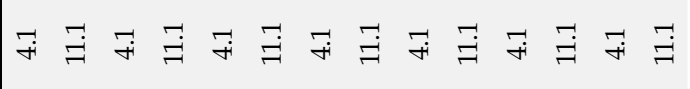 & 0 & $\stackrel{\infty}{=}$ \\
\hline 畣 & 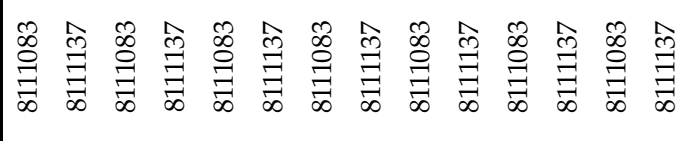 & $\underset{\infty}{\stackrel{\sim}{\overparen{T}}}$ & 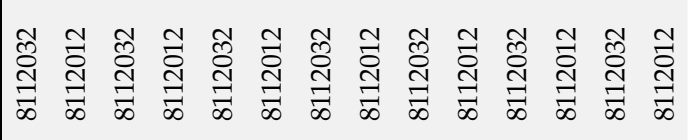 & 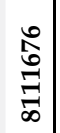 & 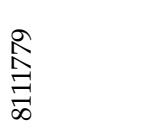 \\
\hline 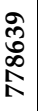 & 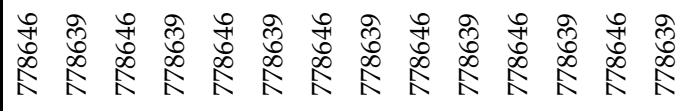 & 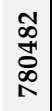 & 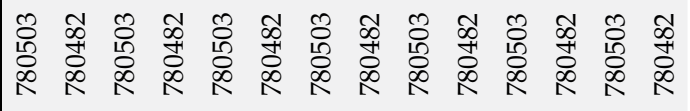 & 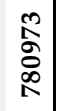 & $\begin{array}{l}\text { 苍 } \\
\stackrel{2}{\infty}\end{array}$ \\
\hline$\infty$ & 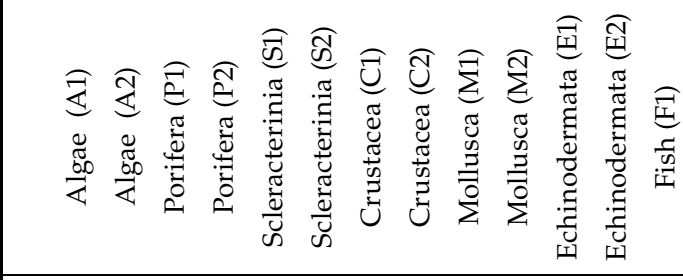 & & 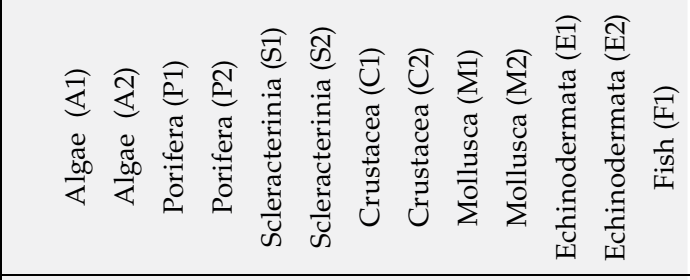 & & \\
\hline 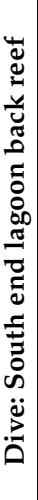 & 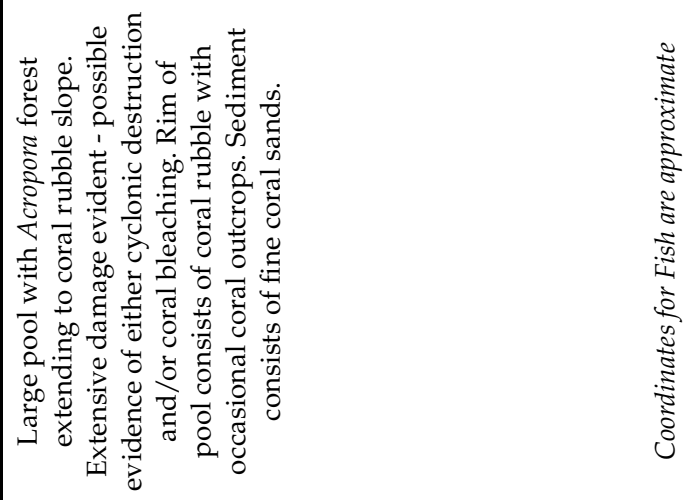 & 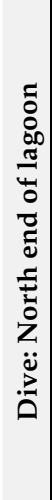 & 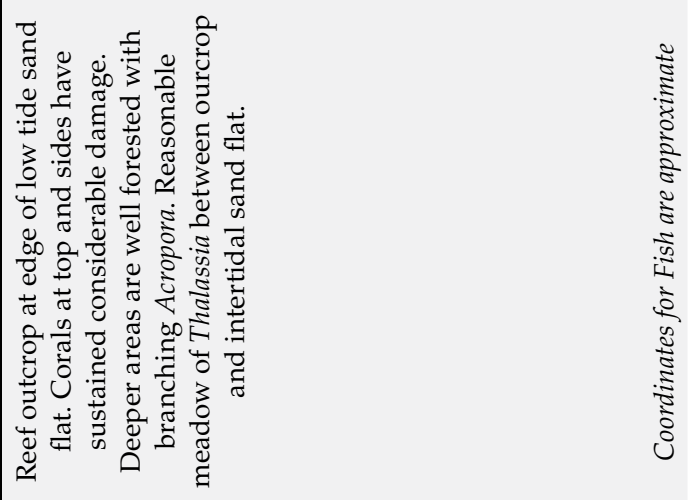 & 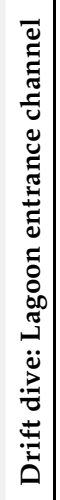 & 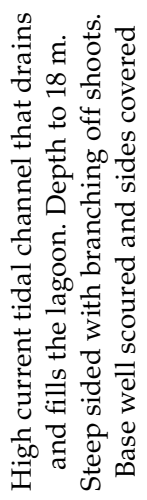 \\
\hline & & & & & \\
\hline
\end{tabular}




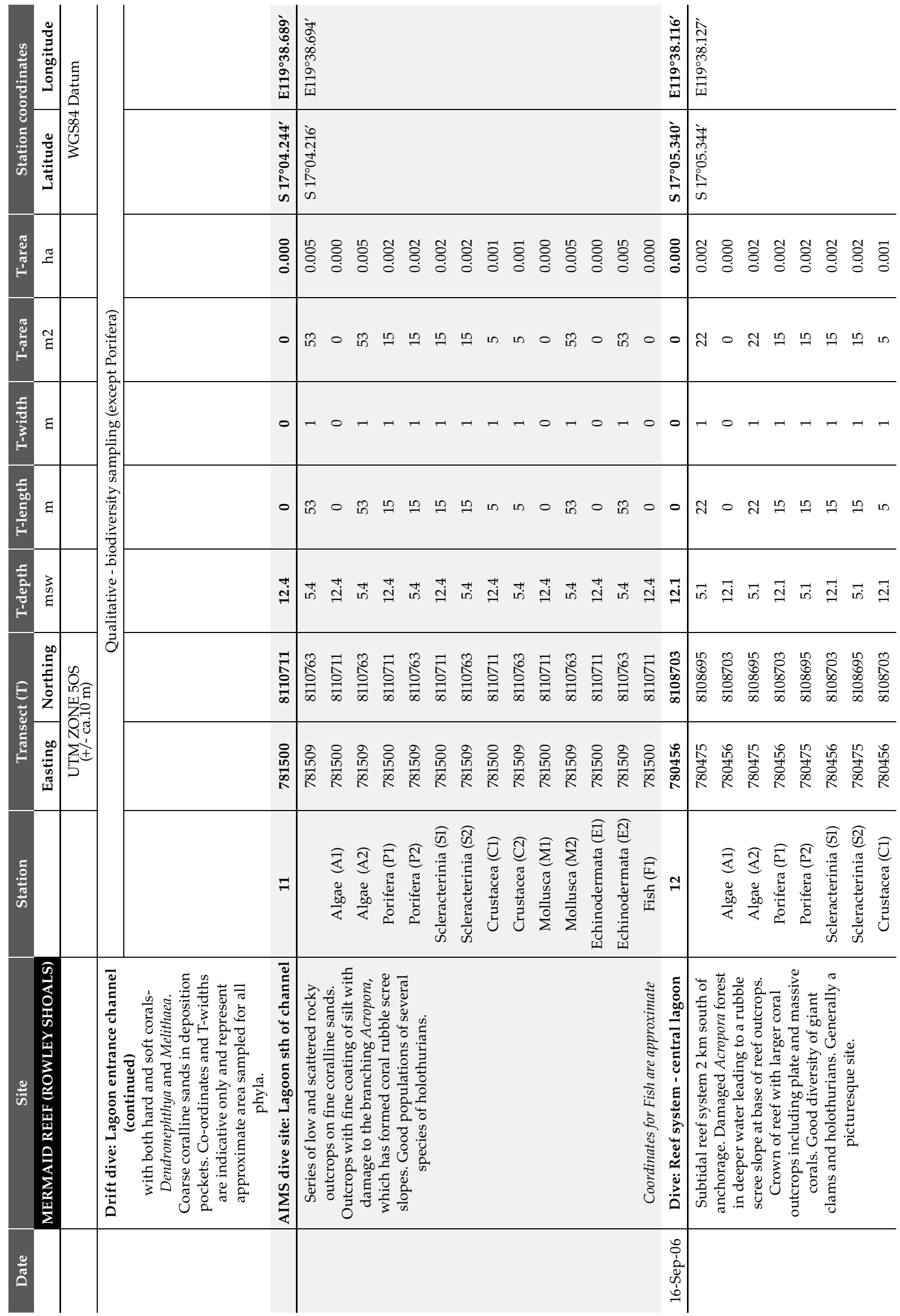




\begin{tabular}{|c|c|c|c|c|c|c|}
\hline & & 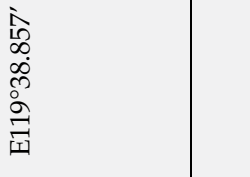 & 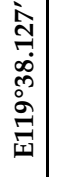 & 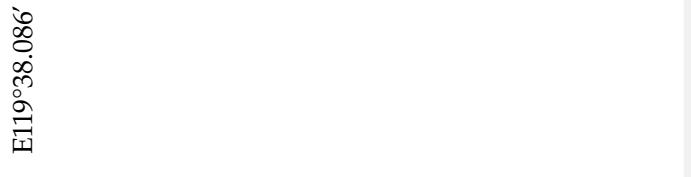 & 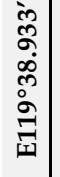 & 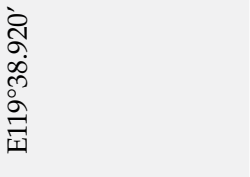 \\
\hline & & $\begin{array}{l}i n \\
\text { in } \\
0 \\
0 \\
0 \\
\text { n } \\
\text { n }\end{array}$ & $\begin{array}{l}1 \\
0 \\
0 \\
o \\
o \\
0 \\
1 \\
\omega \\
\omega\end{array}$ & & 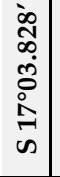 & 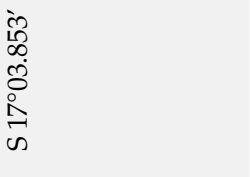 \\
\hline 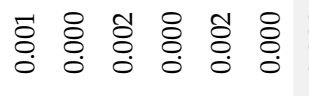 & $\begin{array}{ll}0 \\
\vdots \\
0\end{array}$ & 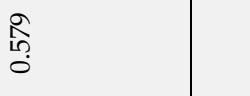 & $\begin{array}{l}8 \\
\vdots \\
0 \\
\dot{0}\end{array}$ & 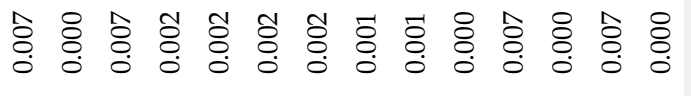 & $\begin{array}{l}: \\
\vdots \\
\vdots \\
0\end{array}$ & 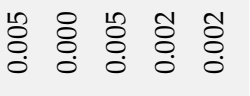 \\
\hline $\operatorname{con} 0$ A 0 & $\circ$ & 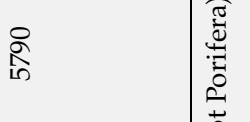 & 0 & Non & 0 & $\vec{n} \circ \vec{n} \stackrel{ }{\sim} \stackrel{ }{\sim}$ \\
\hline$r 0-0 \rightarrow 0$ & 0 & 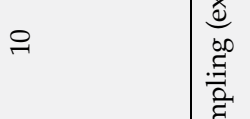 & 0 & 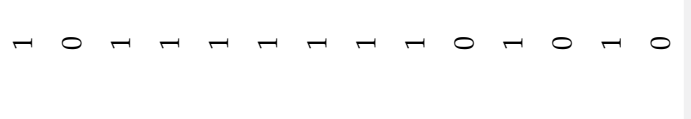 & 0 & $r \quad 0 \quad r-r$ \\
\hline $\ln 04040$ & 0 & 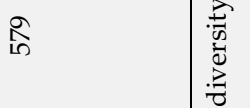 & 0 & Non & 0 & 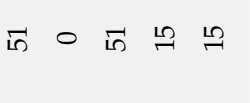 \\
\hline 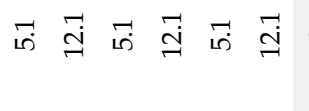 & 0 & 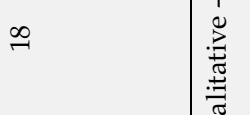 & $\stackrel{\mathscr{g}}{\vec{\theta}}$ & 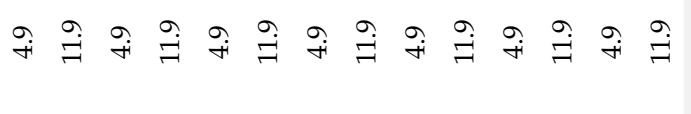 & $\overrightarrow{\mathrm{I}}$ & 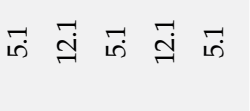 \\
\hline 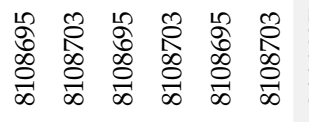 & & $\underset{\infty}{\stackrel{\infty}{\Xi}}$ & $\begin{array}{l}\hat{N} \\
\hat{0} \\
\stackrel{0}{0} \\
\infty\end{array}$ & 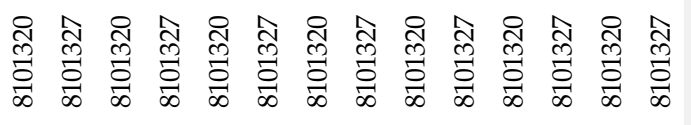 & $\underset{\infty}{\stackrel{N}{\vec{D}}}$ & 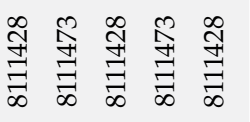 \\
\hline 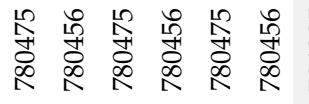 & & 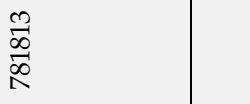 & 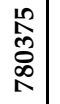 & 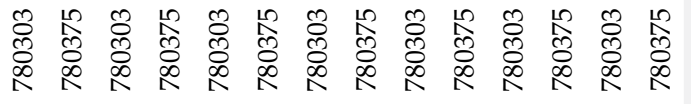 & 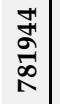 & 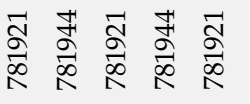 \\
\hline \multirow[t]{3}{*}{ 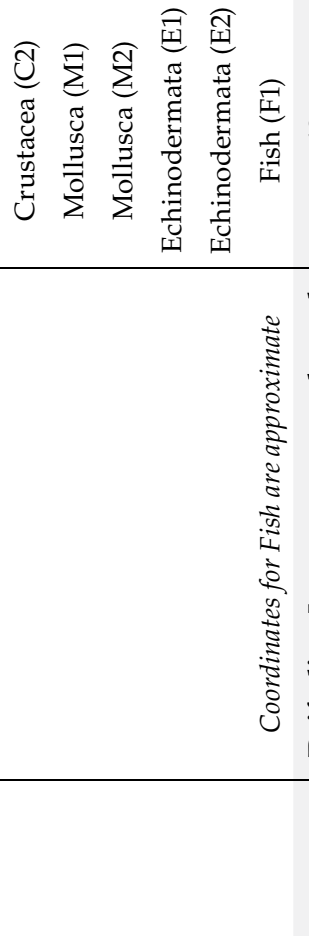 } & & & & 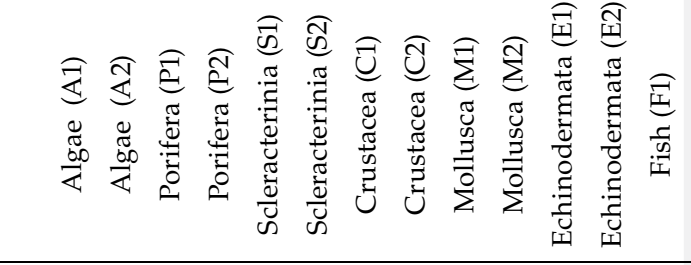 & 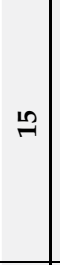 & 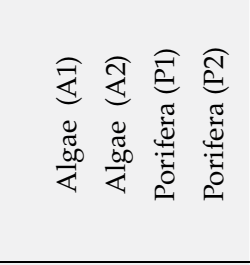 \\
\hline & 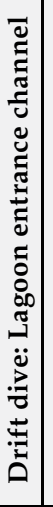 & 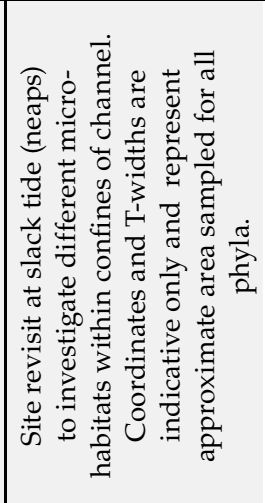 & 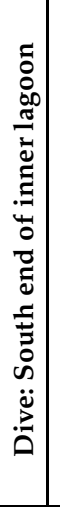 & 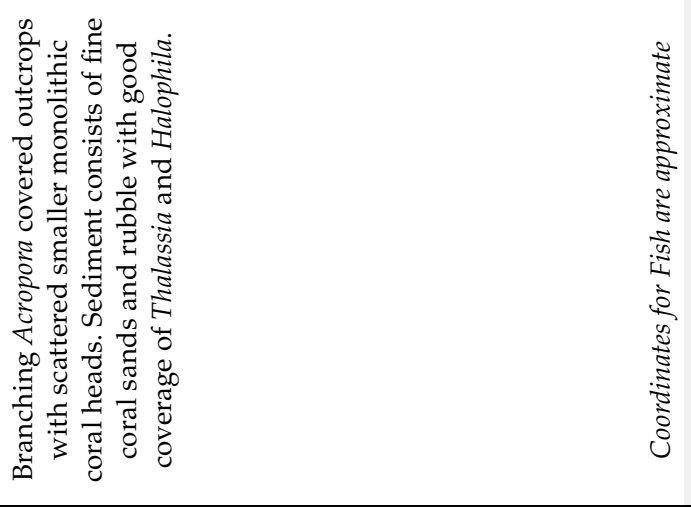 & 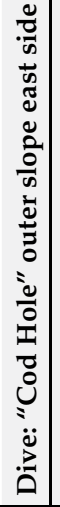 & 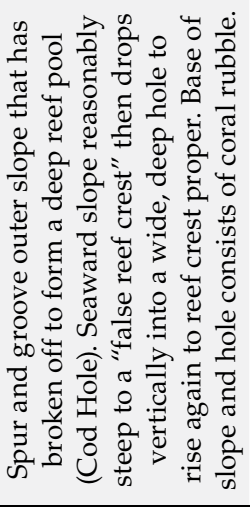 \\
\hline & & & & & 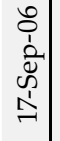 & \\
\hline
\end{tabular}




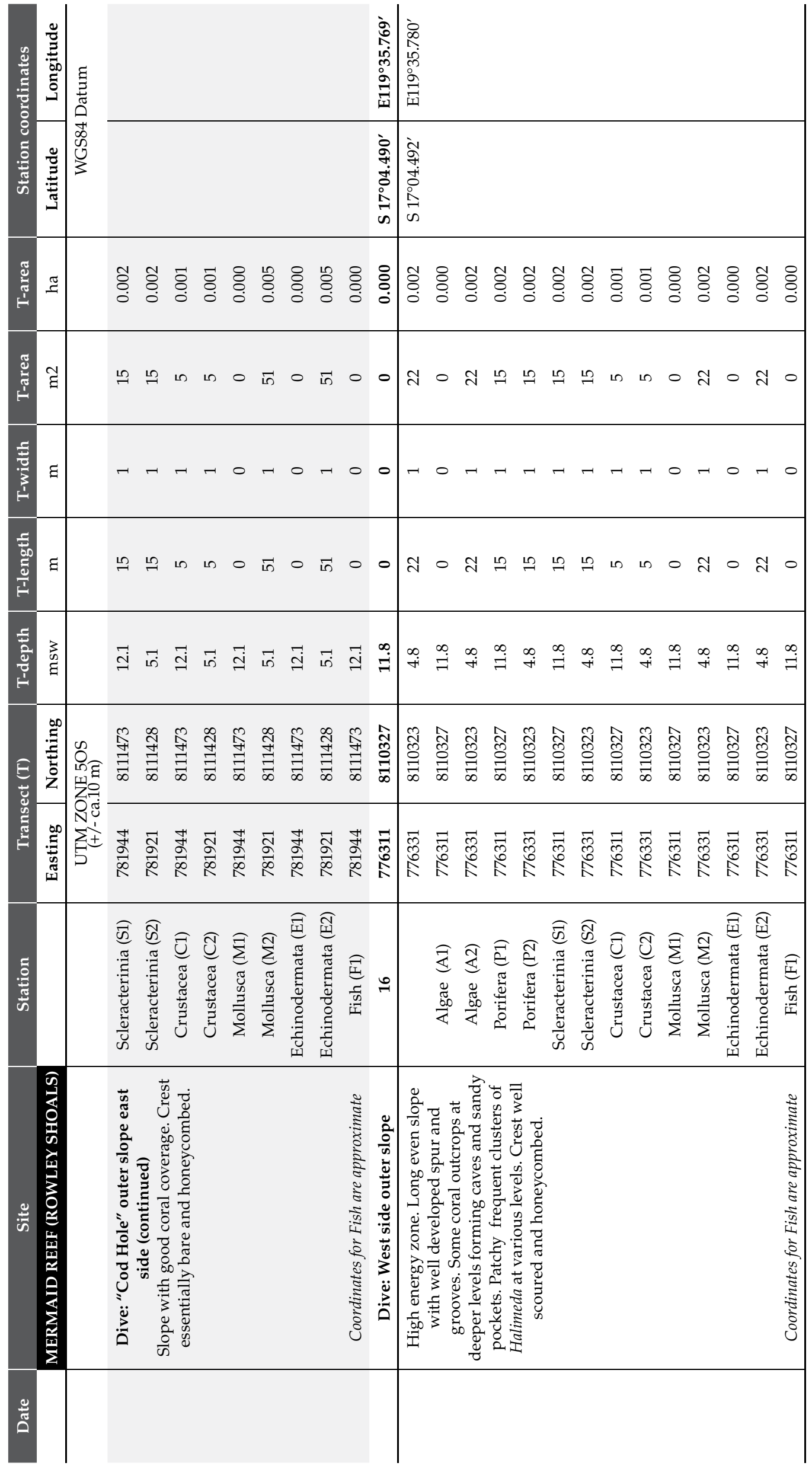




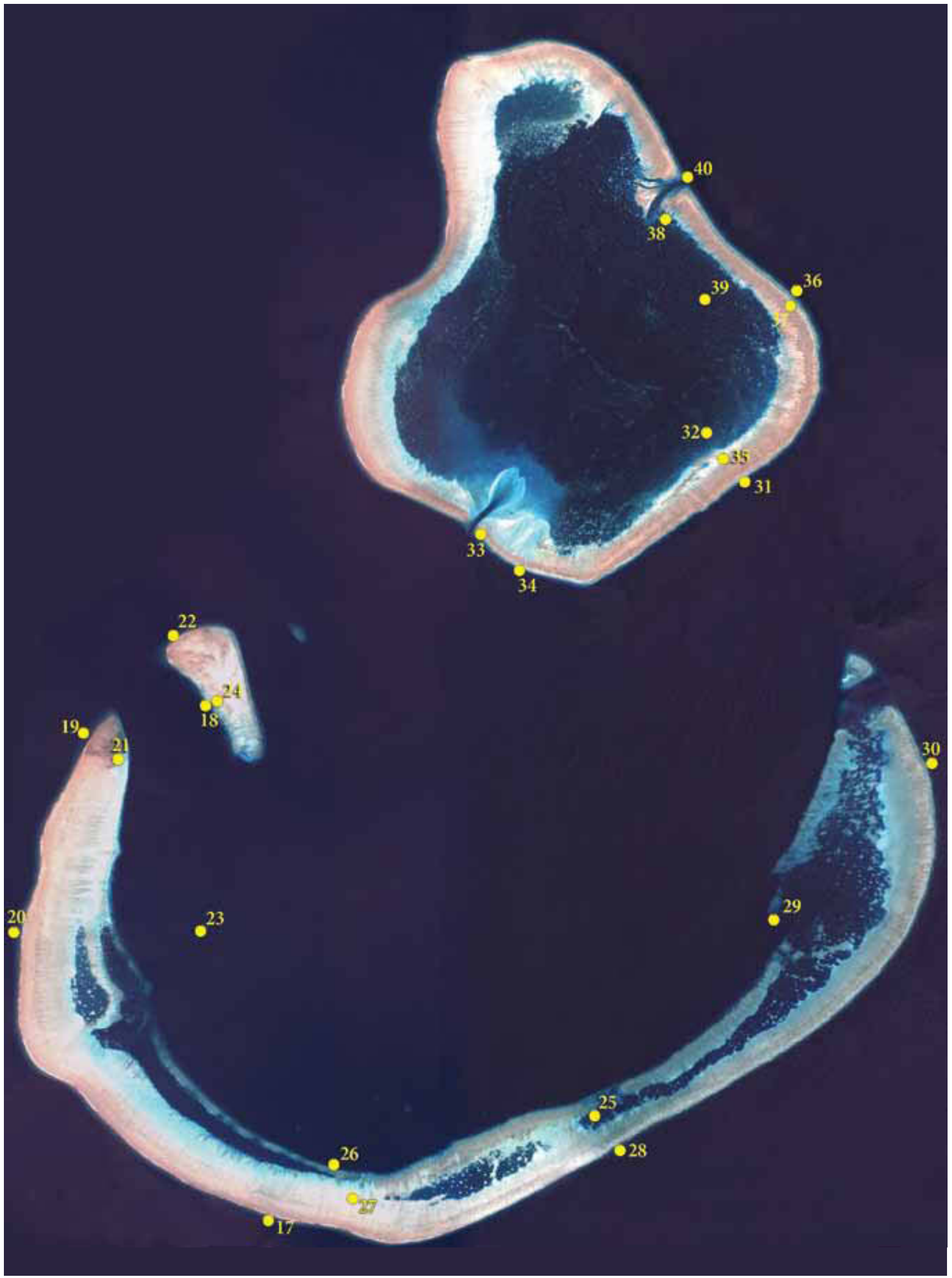

Figure 2 Scott Reef with surveyed station sites (2006) 
ำ

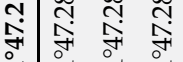

ใู่

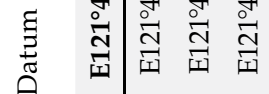

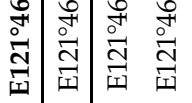

迩

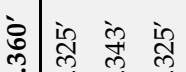

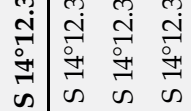

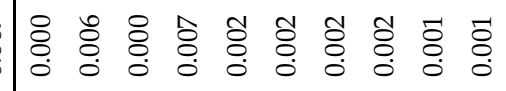

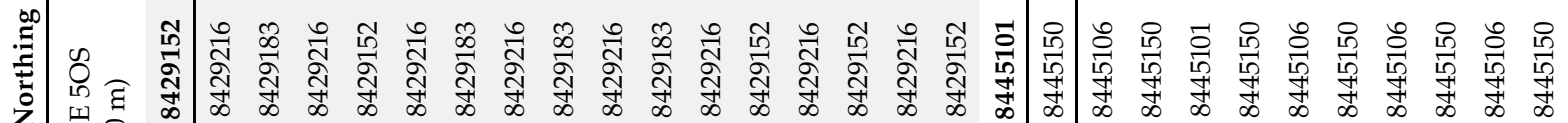

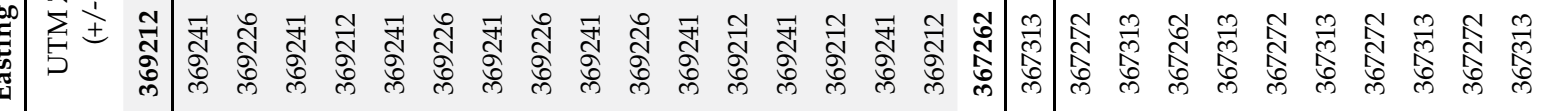

(




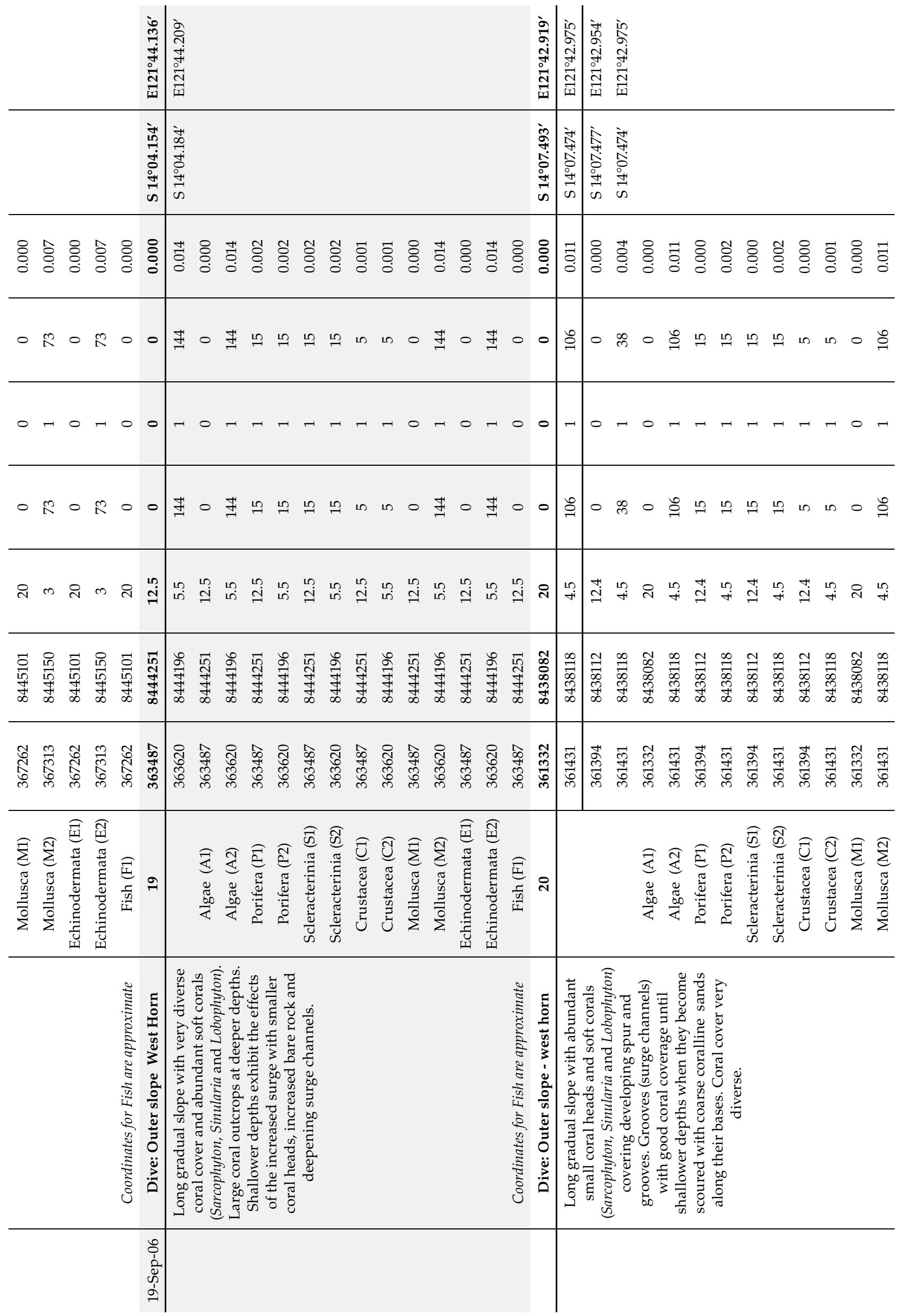




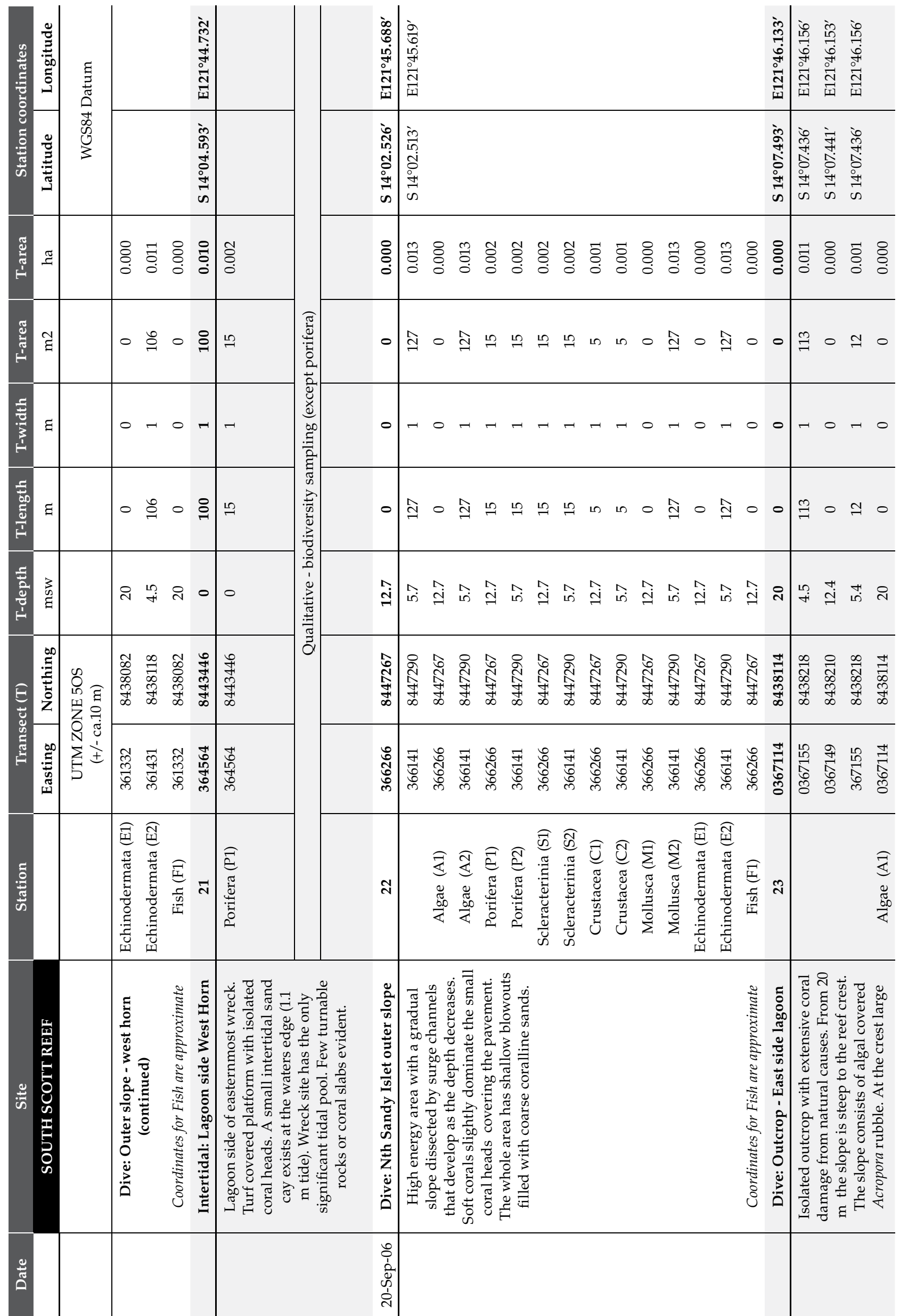




\begin{tabular}{|c|c|c|c|c|}
\hline & 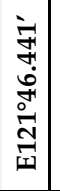 & & 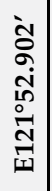 & 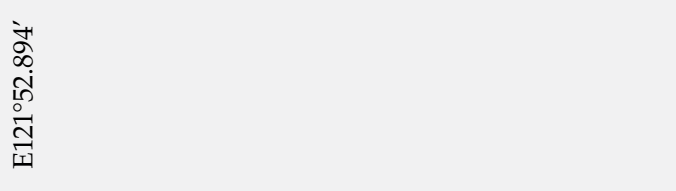 \\
\hline & $\begin{array}{l}\dot{i} \\
0 \\
\dot{0} \\
0 \\
\dot{d} \\
\omega\end{array}$ & & 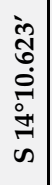 & 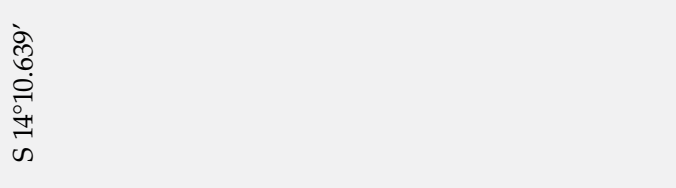 \\
\hline 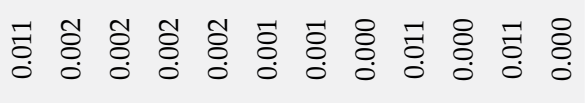 & $\begin{array}{l}0 \\
0 \\
0 \\
0\end{array}$ & $\stackrel{\overbrace{}}{8}$ & $\begin{array}{ll}0 \\
\vdots \\
\vdots \\
\vdots\end{array}$ & 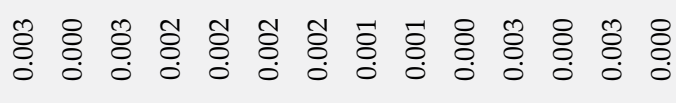 \\
\hline 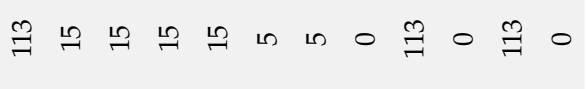 & $\stackrel{一}{2}$ & $\stackrel{2}{\longrightarrow}$ & 0 & 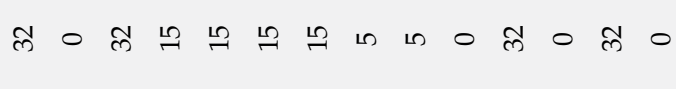 \\
\hline 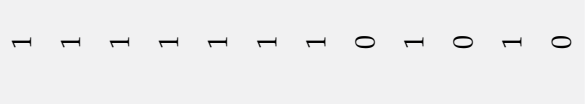 & 7 & $\tau$ & 0 & 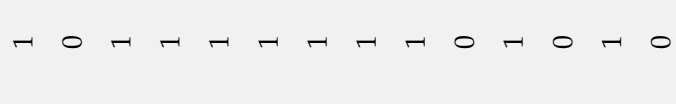 \\
\hline 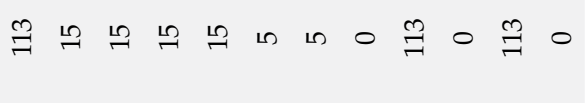 & 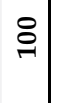 & $\stackrel{2}{2}$ & 0 & लै० \\
\hline 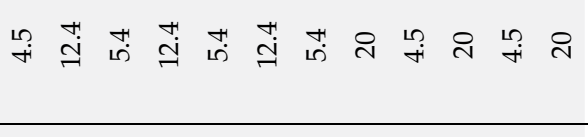 & 0 & 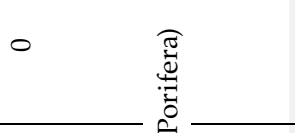 & $\stackrel{\infty}{\stackrel{\infty}{\mathrm{i}}}$ & 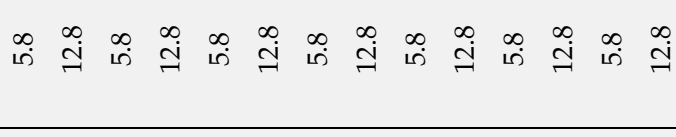 \\
\hline 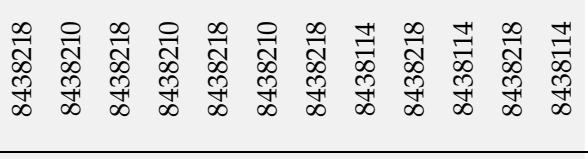 & 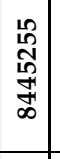 & 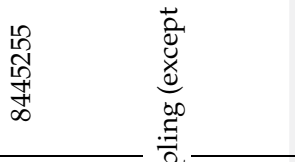 & 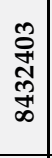 & 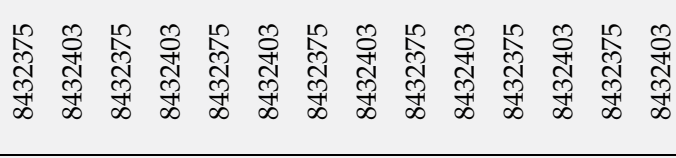 \\
\hline 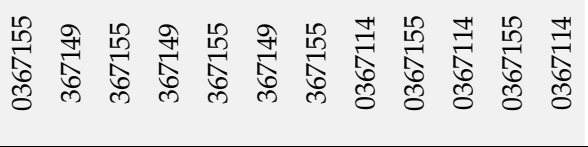 & & 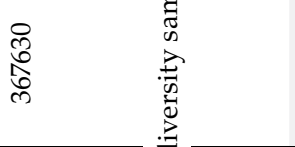 & 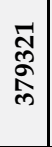 & 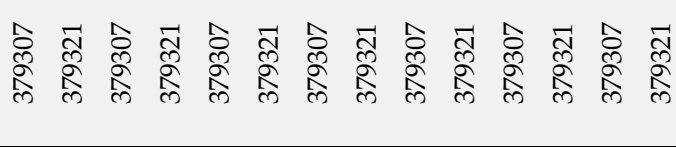 \\
\hline 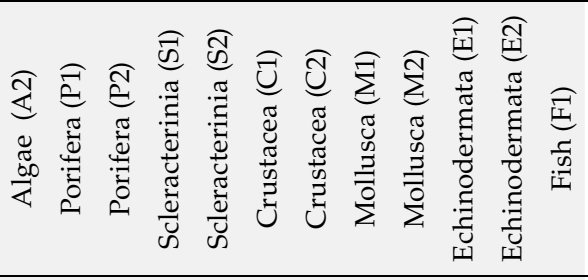 & & a & $\stackrel{\mathscr{2}}{\mathrm{N}}$ & 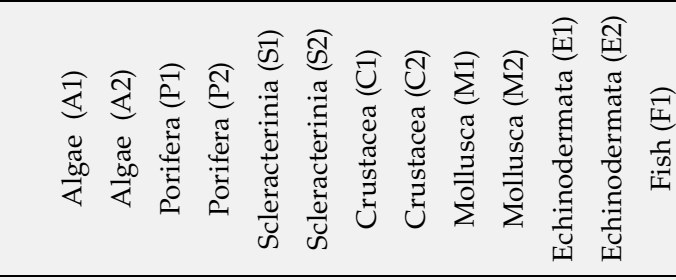 \\
\hline 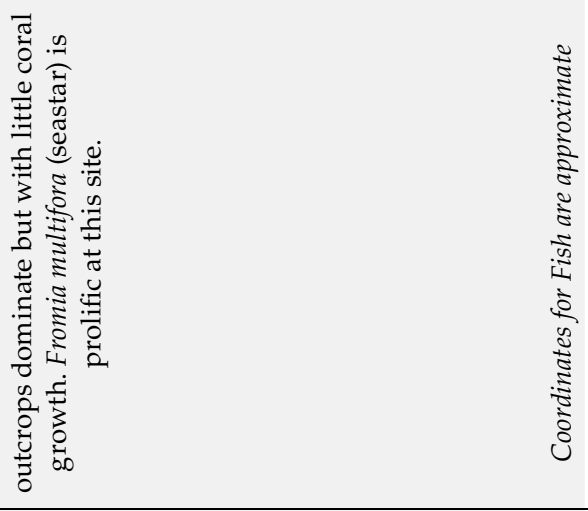 & 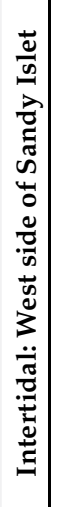 & 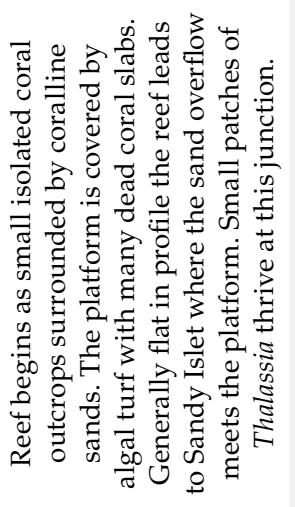 & 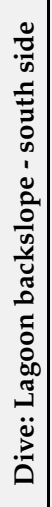 & 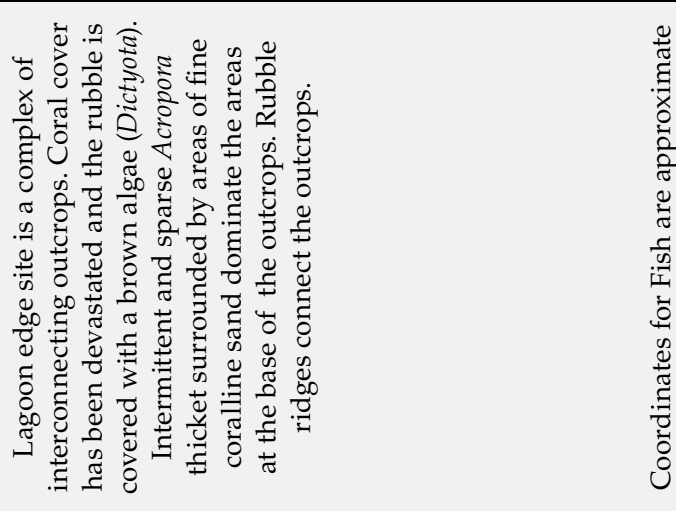 \\
\hline & & & & \\
\hline
\end{tabular}




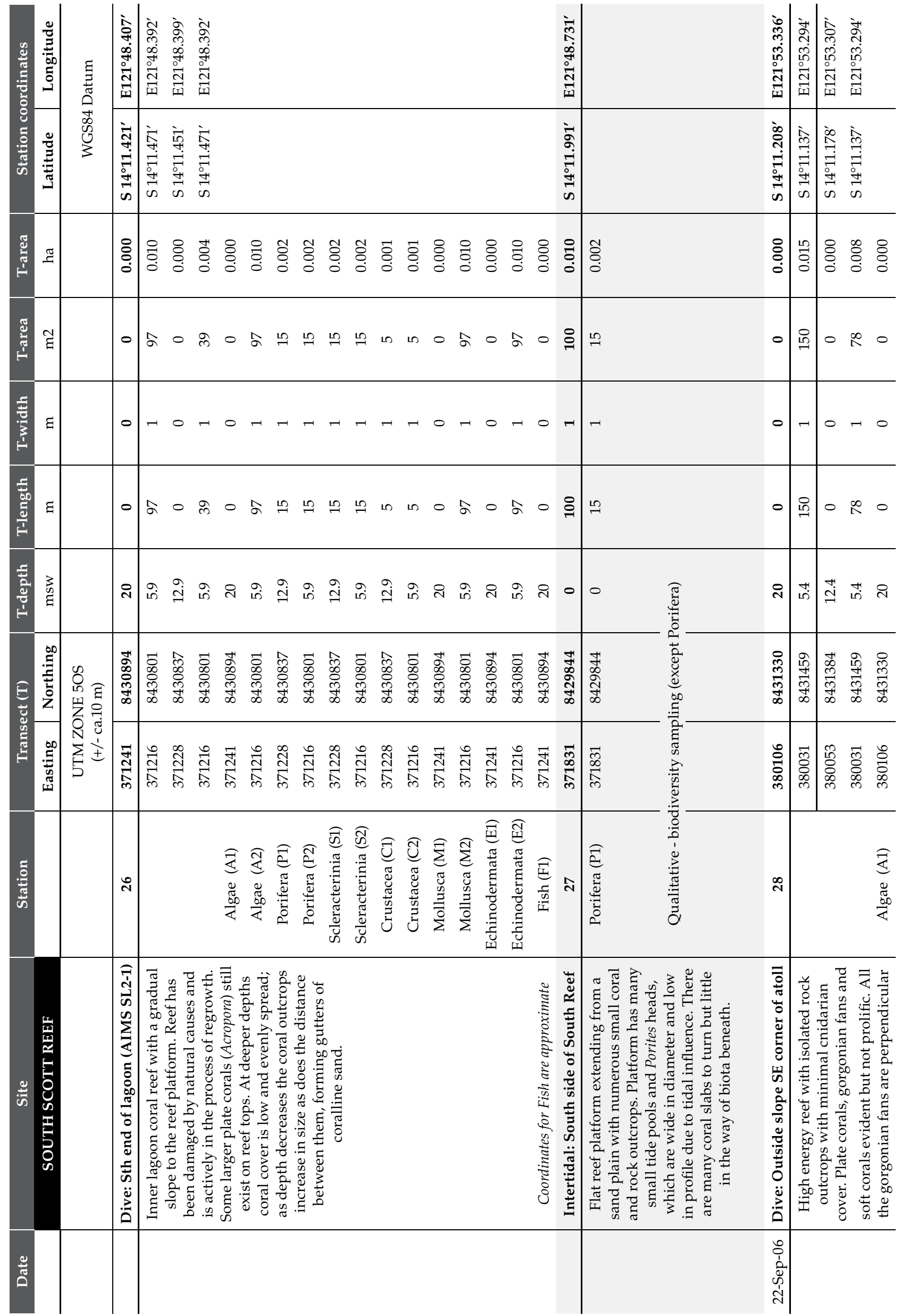




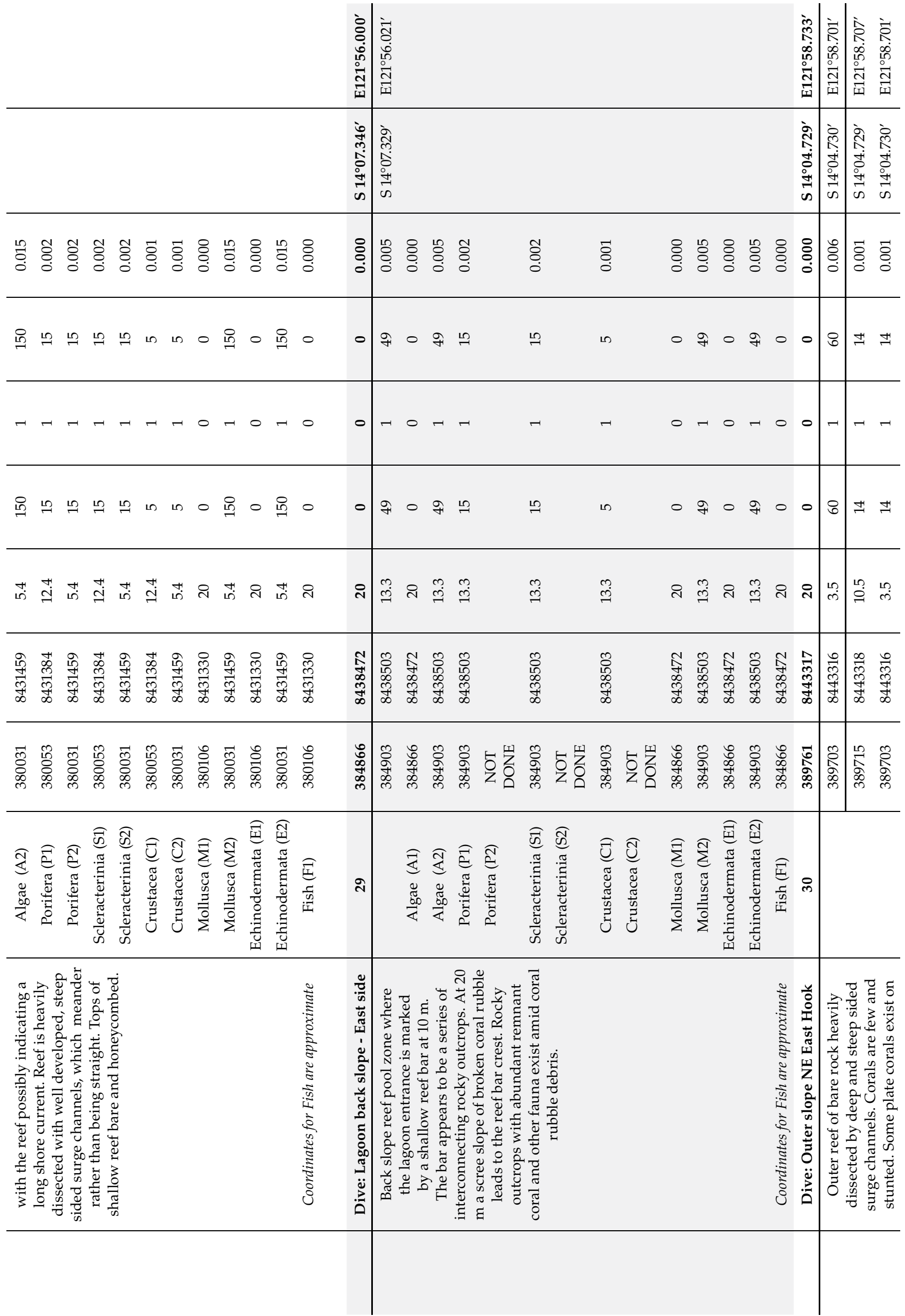




\begin{tabular}{|c|c|c|c|c|c|}
\hline 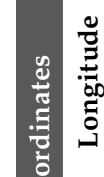 & $\underset{\Xi}{\Xi}$ & & 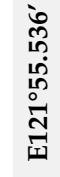 & 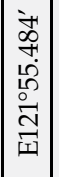 & 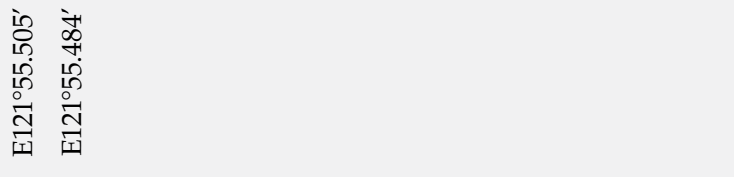 \\
\hline कृ & $\begin{array}{l}\infty \\
\bigcup_{3} \\
\vdots\end{array}$ & & 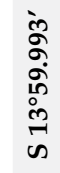 & $\mid \begin{array}{c}i ̀ n \\
\vdots \\
\alpha \\
\sigma \\
o \\
o n \\
\omega \\
\omega\end{array}$ & 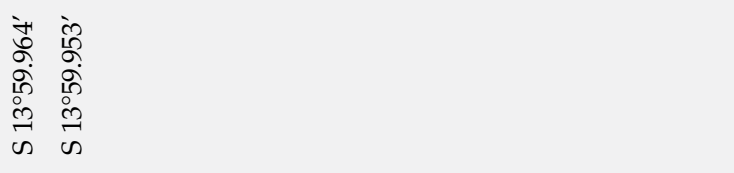 \\
\hline 号 & & 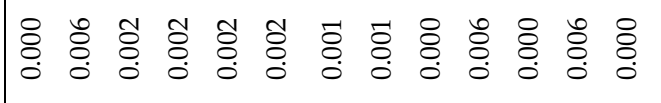 & $\stackrel{0}{\circ}$ & $\begin{array}{l}0 \\
\Xi \\
0\end{array}$ & 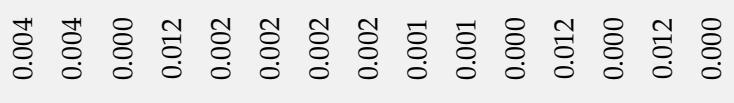 \\
\hline है & & 0 \& 뉵 뉵 & 0 & $\stackrel{\overbrace{}}{\Im}$ & F \\
\hline 象 & & 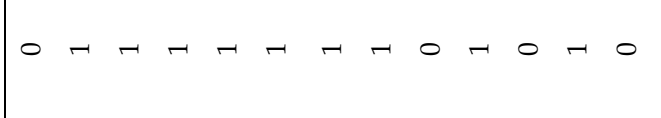 & 0 & -1 & 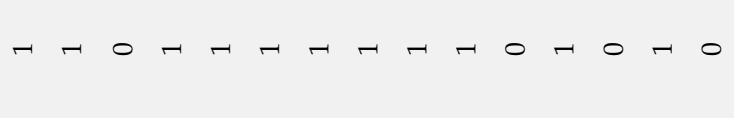 \\
\hline घ & & 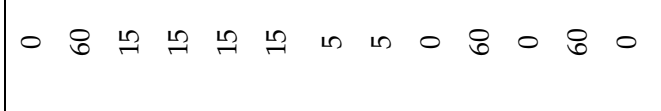 & 0 & $\stackrel{\overbrace{}}{\Im}$ & F \\
\hline 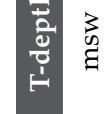 & & 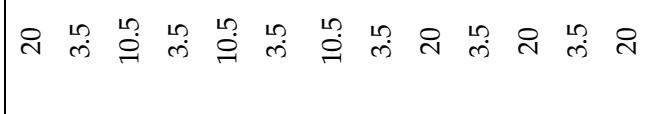 & శి & \begin{tabular}{|l|}
10 \\
$\infty$ \\
$\infty$
\end{tabular} & 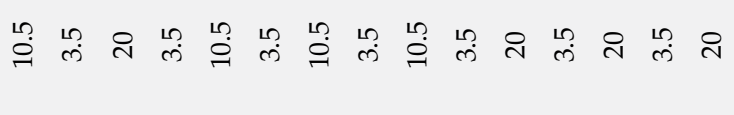 \\
\hline & 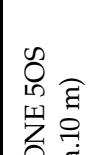 & 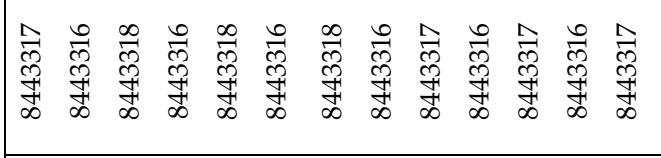 & 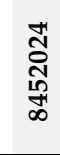 & $\mid$\begin{tabular}{c|c}
$\hat{\alpha}$ \\
$\hat{\alpha}$ \\
के \\
$\infty$
\end{tabular} & 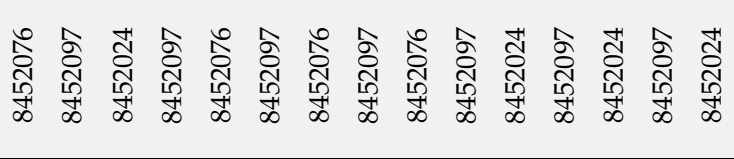 \\
\hline & $\sum_{5}^{\infty} \underbrace{1}$ & 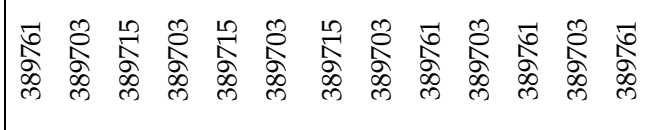 & $\begin{array}{c}0 \\
\stackrel{2}{\infty} \\
\infty \\
\infty\end{array}$ & \begin{tabular}{|l|l}
$n$ \\
$\infty$ \\
$\infty$ \\
$\infty$ \\
$\infty$ \\
$\infty$
\end{tabular} & 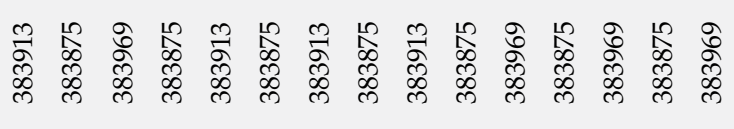 \\
\hline & & 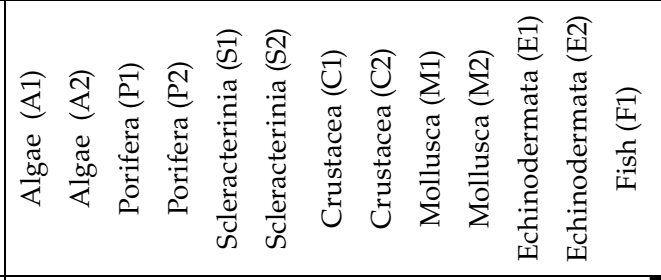 & $\bar{m}$ & & 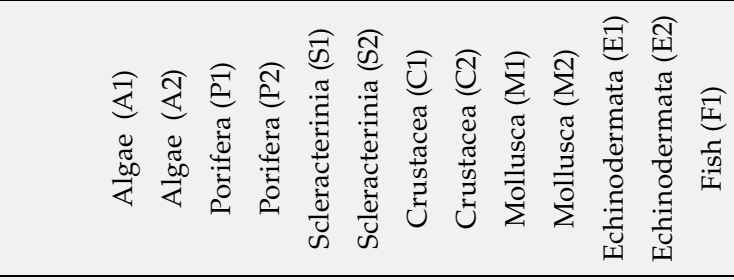 \\
\hline के & & 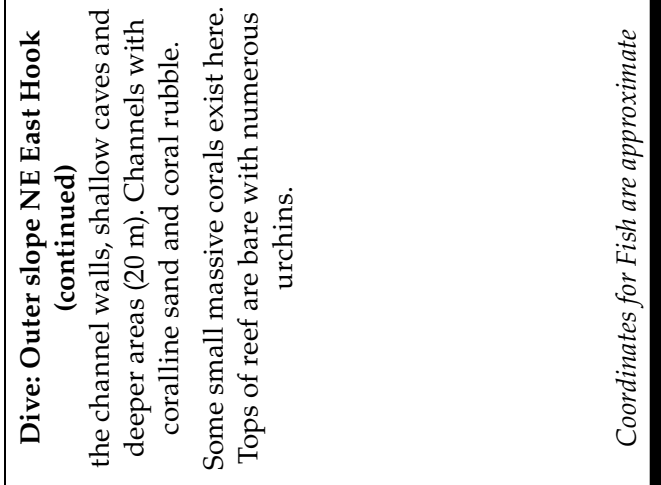 & 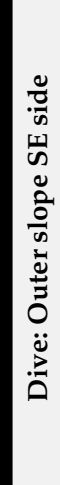 & & 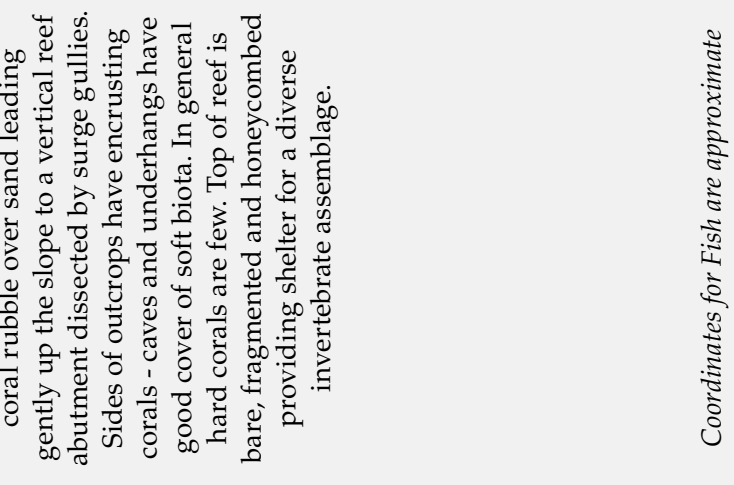 \\
\hline & & & 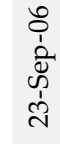 & & \\
\hline
\end{tabular}




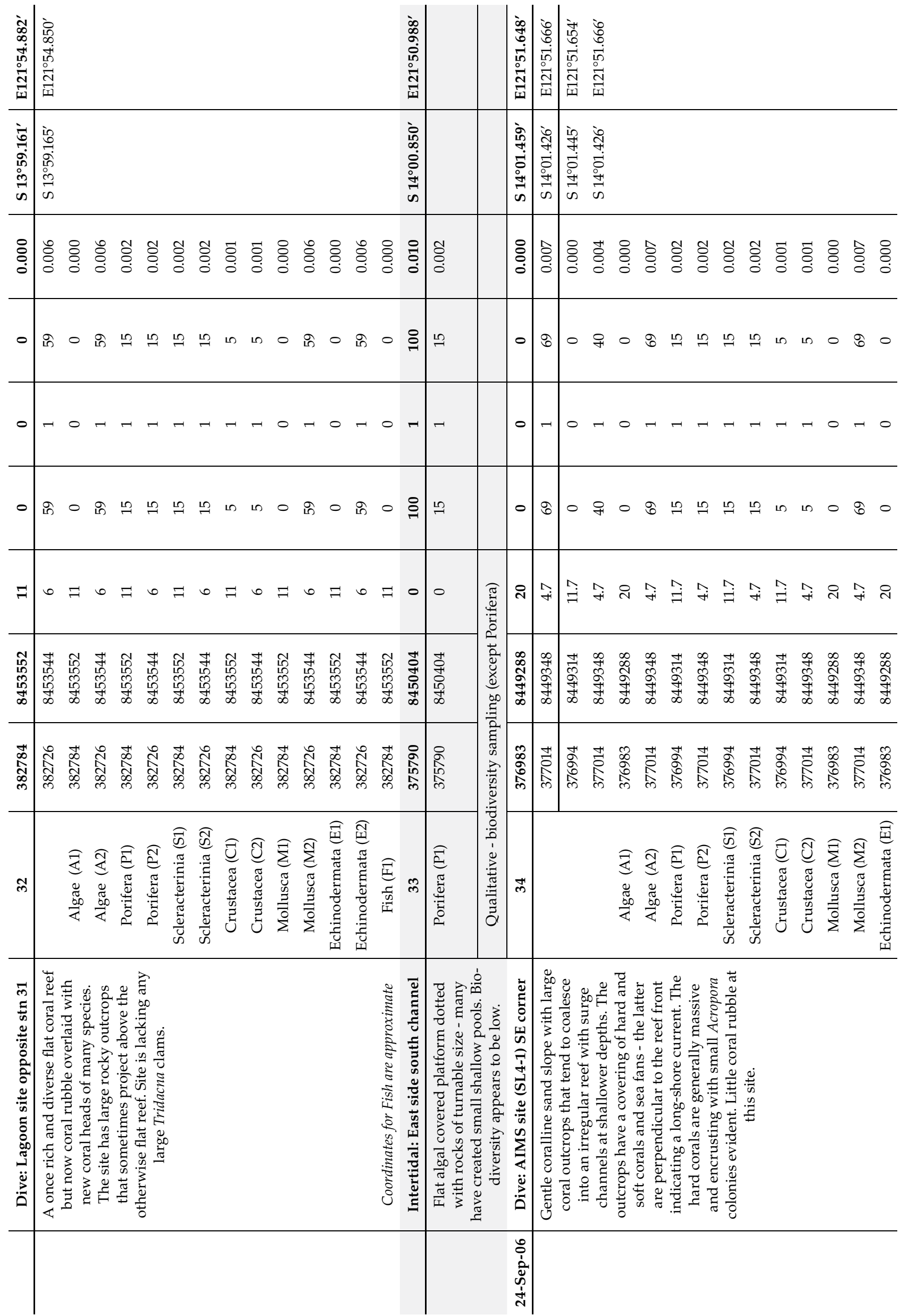




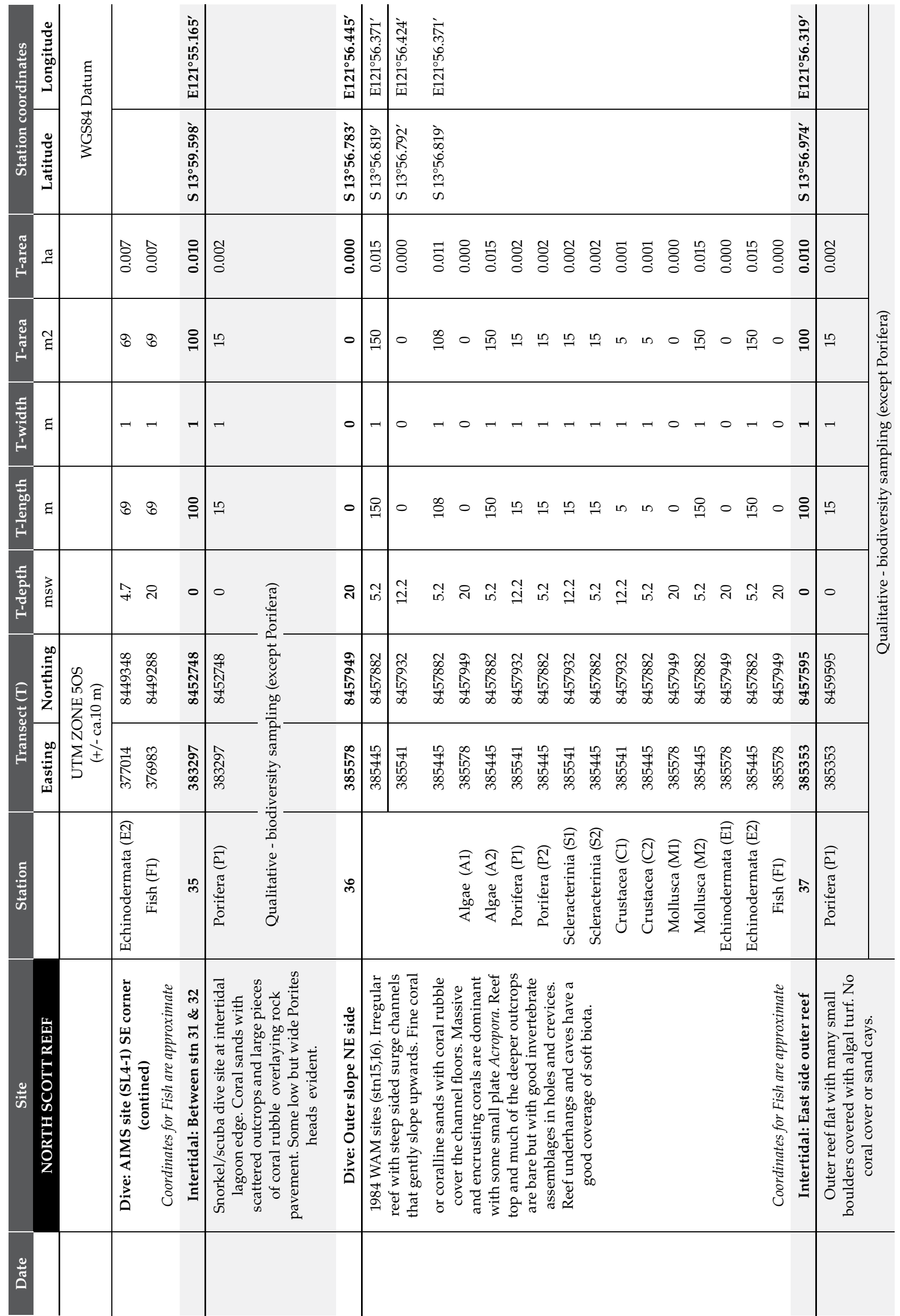




\begin{tabular}{|c|c|c|c|}
\hline & 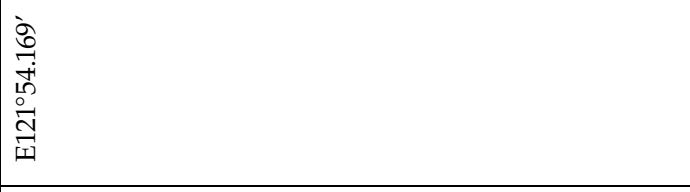 & 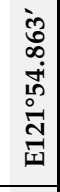 & 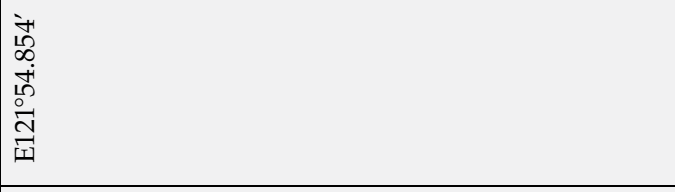 \\
\hline 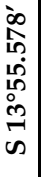 & & 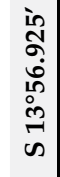 & 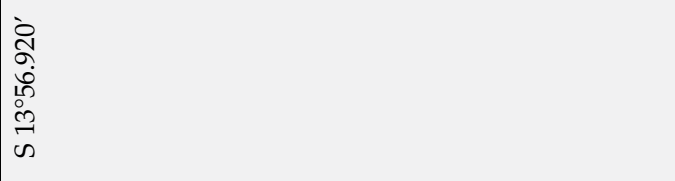 \\
\hline $\begin{array}{l}-10 \\
\vdots \\
0 \\
0\end{array}$ & 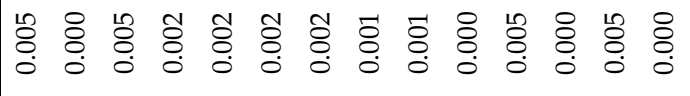 & $\begin{array}{l}0 \\
\stackrel{0}{0} \\
\dot{0}\end{array}$ & 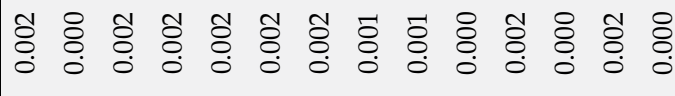 \\
\hline 0 & 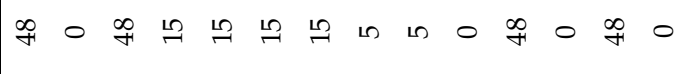 & 0 & तิ 0 กิ \\
\hline 0 & 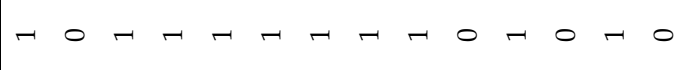 & $\circ$ & 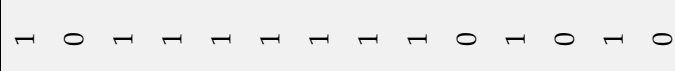 \\
\hline 0 & $\stackrel{\infty}{\circ} \circ \stackrel{\infty}{a}$ ! & 0 & तิ 0 กิ \\
\hline$\stackrel{\vec{g}}{ت}$ & 将芦 & $\stackrel{\sim}{\dot{m}}$ & 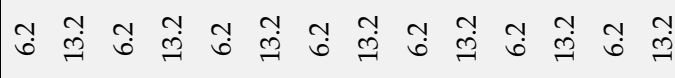 \\
\hline & 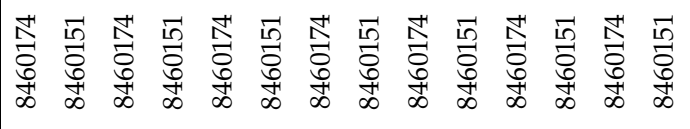 & $\begin{array}{l}0 \\
0 \\
0 \\
1 \\
\vdots \\
\infty \\
\infty\end{array}$ & 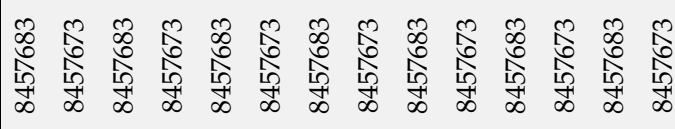 \\
\hline & 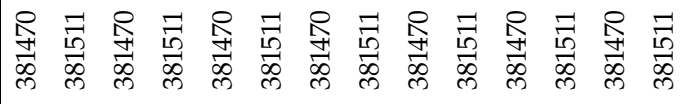 & 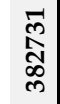 & 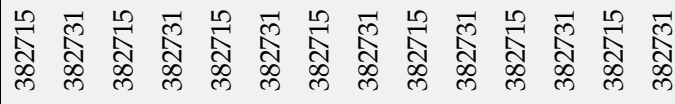 \\
\hline$\stackrel{\infty}{\infty}$ & 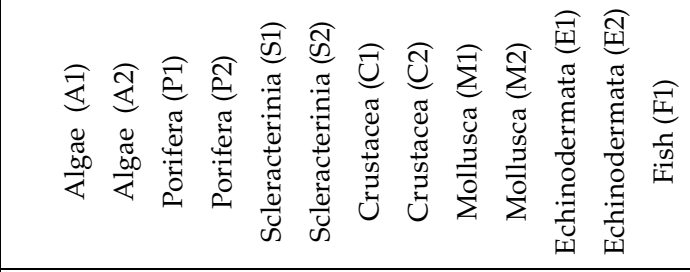 & ले & 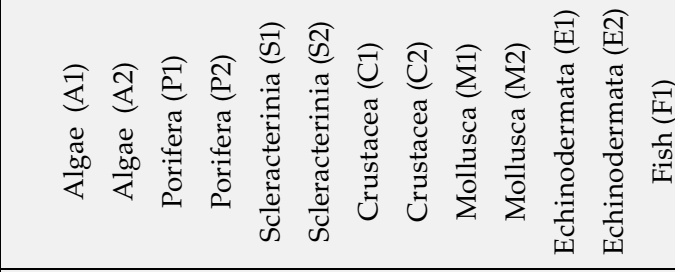 \\
\hline & 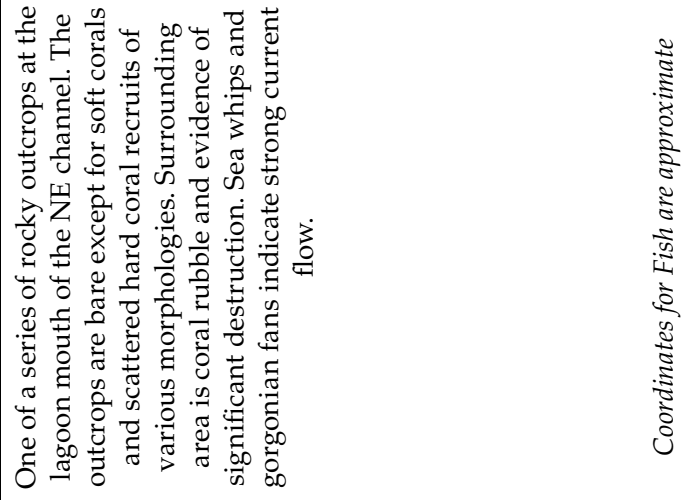 & 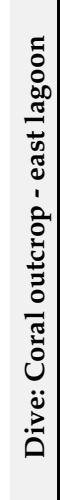 & 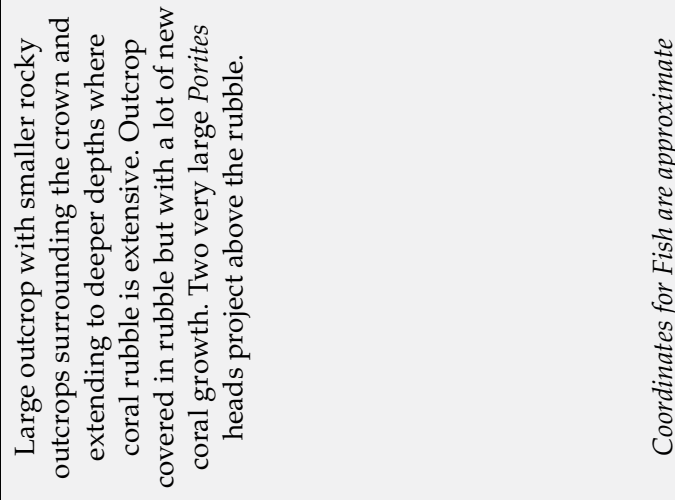 \\
\hline & & & \\
\hline
\end{tabular}



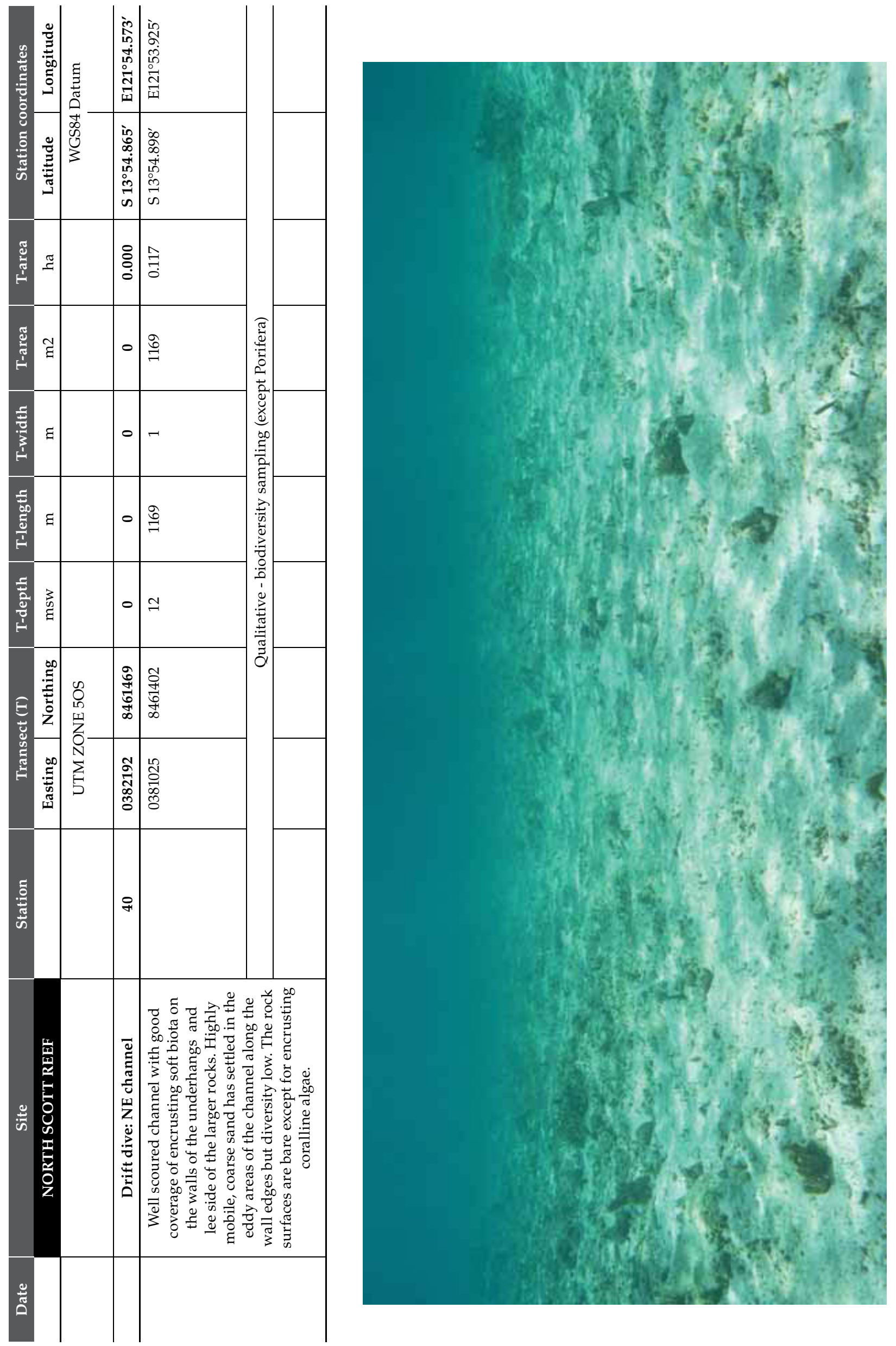


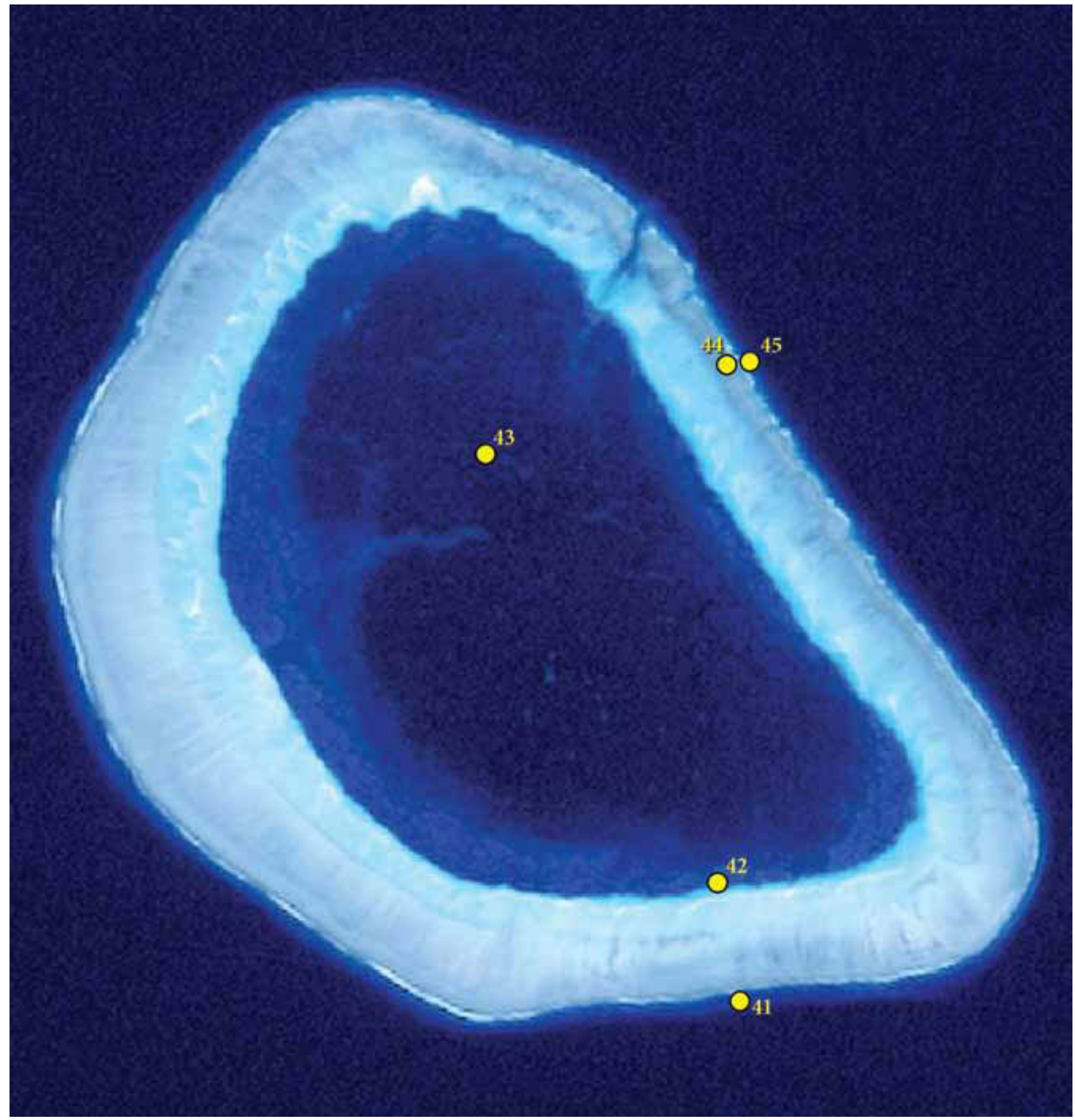

Figure 3 Seringapatam Reef with surveyed station sites (2006) 


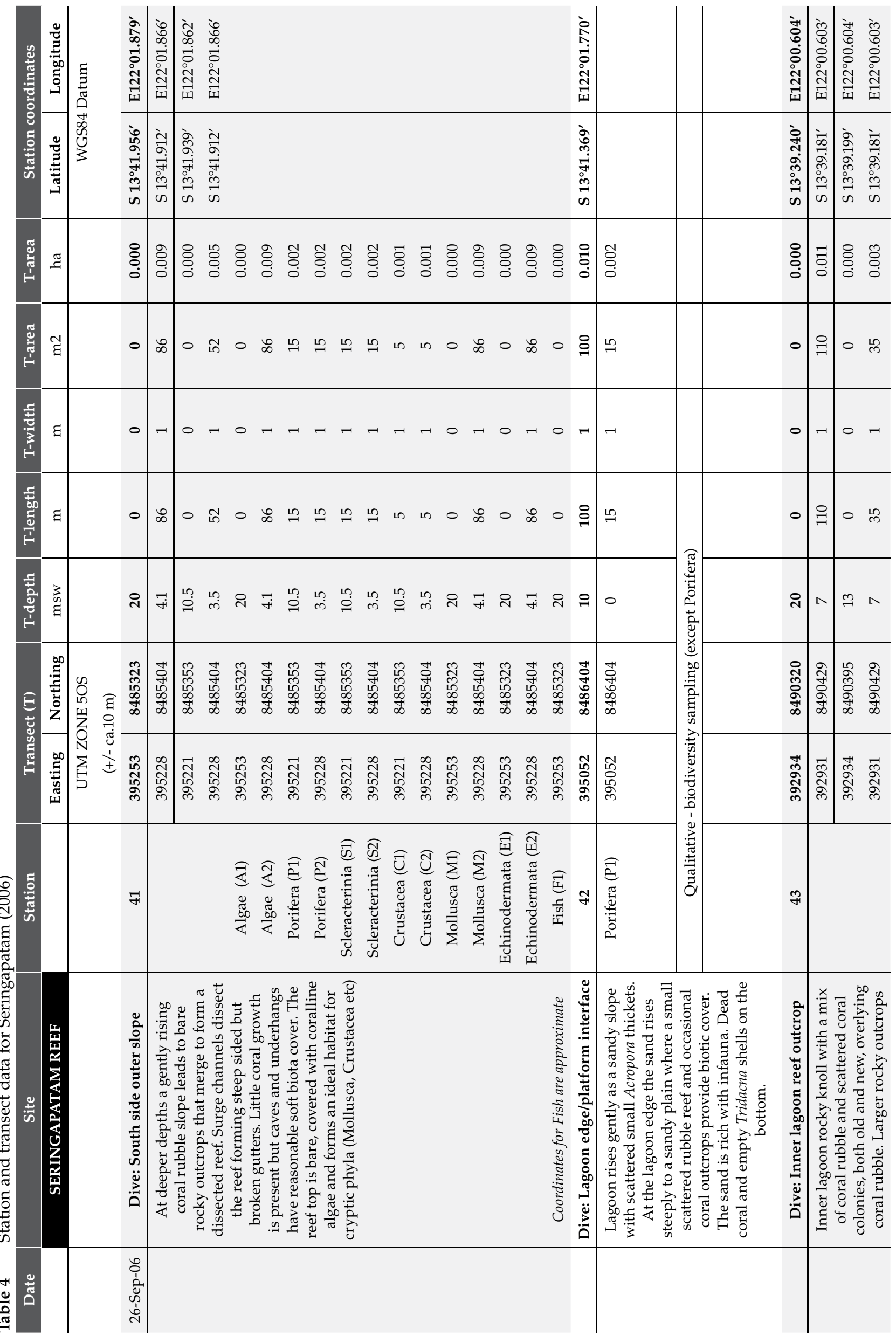




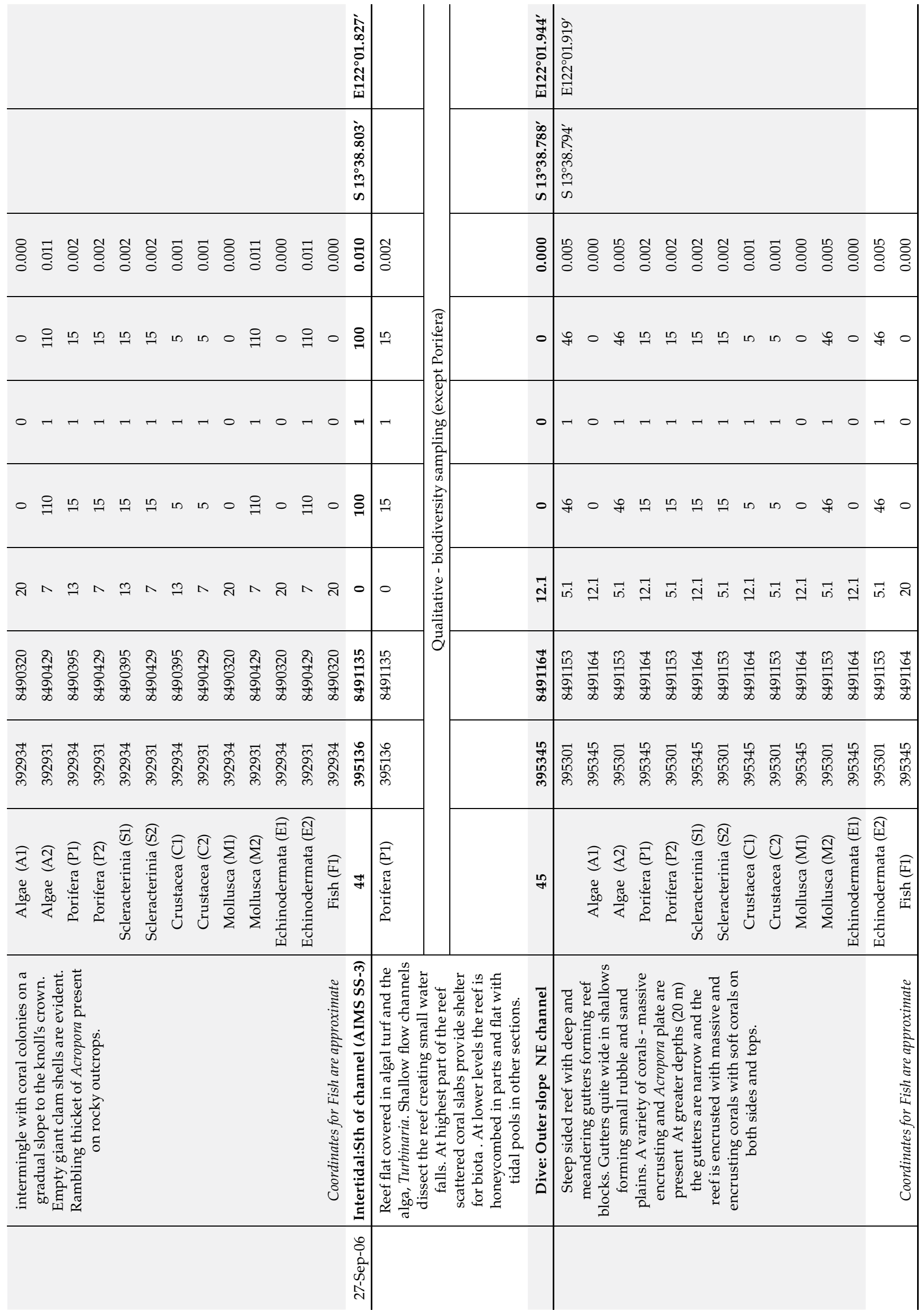


